\title{
Elastic and Viscoelastic Poisson's Ratios: The Theoretical Mechanics Perspective
}

\author{
Harry H. Hilton* \\ Aerospace Engineering Department, College of Engineering and Private Sector Program Division, The National Center for \\ Supercomputing Applications University of Illinois at Urbana-Champaign (UIUC), Urbana, IL, USA \\ Email: h-hilton@illinois.edu
}

How to cite this paper: Hilton, H.H. (2017) Elastic and Viscoelastic Poisson's Ratios: The Theoretical Mechanics Perspective. Materials Sciences and Applications, 8, 291-332.

https://doi.org/10.4236/msa.2017.84021

Received: January 31, 2017

Accepted: April 27, 2017

Published: April 30, 2017

Copyright (C) 2017 by author and Scientific Research Publishing Inc. This work is licensed under the Creative Commons Attribution International License (CC BY 4.0).

http://creativecommons.org/licenses/by/4.0/

Open Access

\begin{abstract}
A recent review publication presented an extensive and comprehensive assessment of the phenomenological relations of Poisson's ratios (PRs) to the behavior and responses of contemporary materials under specific loading conditions. The present review and analysis paper is intended as a theoretical mechanics complement covering mathematical and physical modeling of a single original elastic and of six time and process (i.e. path and stress) dependent viscoelastic PR definitions as well as a seventh special path independent one. The implications and consequences of such models on material characterization are analyzed and summarized. Indeed, PRs based on experimentally obtained 2-D strains under distinct creep and/or relaxation processes exhibit radically different time responses for identical material specimen. These results confirm the PR's implicit path dependence in addition to their separate intrinsic time reliance. Such non-uniqueness of viscoelastic PRs renders them unsuitable as universal material descriptors. Analytical formulations and experimental measurements also examine the physical impossibility of instantaneously achieving time independent loads or strains or their rates thus making certain PR definitions based on constant state variables, while mathematically valid, physically unrealistic and unachievable. A newly developed theoretical/experimental protocol for the determination of the time when loading patterns reach stead-state conditions based on strain accelerations demonstrates the capability to measure this time from experimental data. Due to the process dependent PRs, i.e. stress and stress history paths, the non-existence of a unique viscoelastic PR and of a universal elastic-viscoelastic correspondence principle or analogy (EVCP) in terms of PRs is demonstrated. Additionally and independently, the required double convolution integral
\end{abstract}

*Professor Emeritus of Aerospace Engineering and Senior Academic Lead for Computational Structural/Solid, Mechanics at NCSA. Fellow of the American Institute of Aeronautics and Astronautics (AIAA) and Fellow of the American Society for Composites (ASC). 
construction of linear viscoelastic constitutive relations with the inclusion of PRs is cumbersome analytically and computationally needlessly highly CPU intensive. Furthermore, there is no theoretical fundamental hint as to what loading path is required to produce a unique universal viscoelastic PR definition necessary for formulating a $\mathrm{PR}$ based constitutive relation or an EVCP protocol. The analysis associated with an additional Class VII viscoelastic PR establishes it as a universal representation which is loading path and strain independent while still remaining time dependent. This Class PR can be the one used if it is desired to express constitutive relations in terms of PRs, subject to the caveat applying to all PR Classes regarding the CPU intensiveness in the time space due to triple product and double convolution integral constitutive relations. However, the use PRs is unnecessary since any set of material behavior can be uniquely and completely defined in terms of only moduli and/or compliances. The mathematical model of instantaneous initial loading paths, based on Heaviside functions, is examined in detail and shown to lead to infinite velocities and accelerations. Additionally, even if non-instantaneous gradual loading functions are employed the resulting PRs are still load and load history dependent. Consequently, they represent specialized PR responses applicable and limited to those particular load and history combinations. Although the analyses contained herein are generalized to non-homogeneous linear viscoelastic materials, the main focus is on PR time and process dependence. The non-homogeneous material results and conclusions presented herein apply equally to homogeneous viscoelasticity and per se do not influence the results or conclusions of the analytical development regarding viscoelastic PRs. In short, these PR analyses apply to all linear viscoelastic material characterization.

\section{Keywords}

Elastic and Viscoelastic Poisson's Ratios (PRs), Transient Load Build up, Viscoelasticity, PR Nonlinearities, PR Categories, Loading History PR Dependence

\section{Introduction}

A recent review publication [1] presented an extensive and comprehensive assessment of the phenomenological relations of Poissons ratios (PRs) [2] [3] to the behavior and responses of contemporary materials under specific loading conditions. The present review and analysis paper is intended as a theoretical mechanics complement covering mathematical and physical modeling of a single original elastic and of six time and process (i.e. path and stress) dependent viscoelastic PR definitions as well as a seventh special path independent one.

Viscoelasticity had its origins with the seminal studies of Kelvin, Maxwell, Voigt, Boltzmann and Volterra during the period of 1865 to 1913 [4]-[11]. However, it did not start to flourish until the mid 1940s when general constitutive relations and elastic/viscoelastic correspondence principles (EVCP) were 
starting to be developed [12]-[17]. These works were followed by the works in the partial listings of Refs. [18]-[78]. There remain two major contentious issues regarding viscoelastic PRs [79]-[104] and both, while summarized in this section, are analyzed and discussed in detail subsequently.

1) $P R$ dependence on time and on stress and/or strain history

These are two separate phenomena. For instance, in linear viscoelasticity moduli, compliances, relaxation and creep functions are only time dependent, while all PRs except Class VII are both implicitly time dependent and stress history (path) dependent. The time dependence of viscoelastic PRs was firmly established experimentally over twenty years ago [79] and yet archival journals continue to publish analyses based on constant viscoelastic PRs. See [85]-[91] for a partial list of such references. For over half that period, it has been analytically established that these same viscoelastic PRs are not only time dependent but also functions of stresses and stress histories. Thus making them non-unique quantities unsuited for viscoelastic material descriptors [85]-[91]. Additionally, as has been analytically proven that some PR classes fail to produce expressions in terms of PRs leading to comprehensive elastic-viscoelastic correspondence principles (EVCPs) [14] [16]. Also see Table 1. This is in contrast to isotropic and anisotropic linear viscoelastic constitutive relations based on moduli or compliances, but without PRs, which under the same conditions and for identical materials indeed rise to produce EVCPs in their own right.

\section{2) Contributions of the loading phase in determining material properties}

The importance of starting loading phases on subsequent viscoelastic responses and consequently on material characterization (moduli/compliances) has been demonstrated through analytical simulations in [95], [96] and through experimental investigations in [97]-[104]. Less restrictive conclusions based on the "en-times-rule" have been reached in [105] [106] [107] [108] based on comparisons of strain responses. While such approximations may be satisfactory for long time stress analyses, the neglect of loading phases produces significant errors in viscoelastic characterizations [95] [96]. In [53] it is stated that measurements of the loading times $t_{1}$ are subject to errors with larger errors corresponding to shorter $t_{1}$ times. The analysis is based on constant stress loadings without inclusion of loading cycles in the data analysis.

In [109] and [110] the loading cycles are taken into account when experimentally determining Class I PRs. References [111] [113] [114] [115] are recent examples of experimental viscoelastic PR characterizations. These references, as well as [1] and [87] [88] [90], include extensive bibliographies that are not repeated here. However, these publications as well as others do not take into account the significant contributions and specializations of PR loading path dependences.

Finally, it is important to note that experimental determinations of viscoe- 
Table 1. Viscoelastic Poisson's ratios and the EVCP.

\begin{tabular}{|c|c|c|c|c|}
\hline Class & Name & Viscoelastic & $\begin{array}{c}\text { Path } \\
\text { Dependent }\end{array}$ & EVCP \\
\hline & & Poisson's Ratio $(\boldsymbol{i} \neq \boldsymbol{j}, \boldsymbol{j} \neq 1)$ & pendent & \\
\hline I & Original [2] [3] & $v_{i j}^{I}(x, t) \underline{\underline{\operatorname{def}}}-\frac{\epsilon_{i j}(x, t)}{\epsilon_{i i j}(x, t)}$ & YES & $\mathrm{NO}^{\mathrm{I}}$ \\
\hline \multirow[t]{2}{*}{ II } & $\epsilon_{i i}(x)$ only & $v_{i j}^{I I}(x, t) \underline{\underline{\operatorname{def}}}-\frac{\epsilon_{i j}(x, t)}{\epsilon_{i i j}(x, t)}$ & \multirow[t]{2}{*}{ YES } & $\begin{array}{l}\text { YES, but } \\
\text { limited }\end{array}$ \\
\hline & 1-D [29] [64] & $(\boldsymbol{i} \neq \boldsymbol{j}, \boldsymbol{j} \neq 1)$ & & $\begin{array}{c}\text { to } \epsilon_{11}(x) \\
\text { only }\end{array}$ \\
\hline III & $\begin{array}{c}\text { LT or FT }[29] \\
{[87]}\end{array}$ & $\overline{\bar{v}}_{i j}^{I I I}(x, \omega) \underline{\underline{\operatorname{def}}}-\frac{\overline{\bar{\epsilon}}_{i j}(x, \omega)}{\overline{\overline{\bar{\epsilon}_{i i}}}(x, \omega)}$ & YES & YES \& NO ${ }^{I I}$ \\
\hline IV & Hencky [75] & $v_{i j}^{I V}(x, t) \underline{\underline{\operatorname{def}}}-\frac{\log \left[1+\epsilon_{\underline{i j}}(x, t)\right]}{\log \left[1+\epsilon_{i \underline{i}}(x, t)\right]}$ & YES & $\begin{array}{c}\mathrm{NO}, \\
\text { nonlinear }^{\mathrm{IV}}\end{array}$ \\
\hline V & Velocity [76] & $\frac{\partial v_{i j}^{V}(x, t)}{\partial t} \underline{\underline{\operatorname{def}}}-\frac{\frac{\partial \epsilon_{i j}(x, t)}{\partial t}}{\frac{\partial \epsilon_{i i}(x, t)}{\partial t}}$ & YES & $\mathrm{NO}^{\mathrm{V}}$ \\
\hline VI & $\sigma_{11}(x)$ only & $v_{1 j}^{V I}(x, t) \underline{\underline{\text { def }}}-\frac{C_{\underline{i j 11}}(x, t) \sigma_{11}(x)}{C(x, t) \sigma_{11}(x)}$ & YES & $\begin{array}{l}\text { YES, but li- } \\
\text { mited }\end{array}$ \\
\hline \multirow{3}{*}{ VII } & $1-\mathrm{D}[90]$ & See Note VI below. & \multirow{3}{*}{ NO } & $\begin{array}{c}\text { to } \sigma_{11}(x) \\
\text { only }^{\mathrm{VI}}\end{array}$ \\
\hline & $\begin{array}{l}\text { pseudo } \\
\text { PR not }\end{array}$ & $\overline{\bar{v}}_{1 j}^{V I I}(x, \omega) \underline{\underline{\text { def }}}-\frac{\overline{\bar{C}}_{11 j i j}(x, \omega)}{\overline{\bar{C}}(x, \omega)}$ & & \multirow[t]{2}{*}{$\mathrm{YES}^{\mathrm{VII}}$} \\
\hline & $\begin{array}{c}\text { based on } \\
\text { strains }\end{array}$ & & & \\
\hline
\end{tabular}

NOTES: All PRs depend on time, stresses and stress-time histories, and these results apply equally to homogeneous and nonhomogeneous media. None of the PRs represent universal material properties as they are path dependent, i.e. $v_{i j}(x, t) \equiv v_{i j}\left[x, t, \sigma_{i i}(x, t)\right] i \neq j \quad$ I Not in proper form in FT space for EVCP. See Table 3. II See the Section "The physics of time invariant stresses, etc." for the physical difficulties associated with achieving constant strains or stresses or constant rates for $0 \leq t \leq \infty$. III Inverse FT has double convolution integral in $t$ space. See Eqs. (32) for some physically unattainable forms, which do not lead to EVCP. Iv Based on natural strains and, therefore, nonlinear. v Similar EVCP difficulties as Case I. vI If and only if $\int_{-\infty}^{t} C_{i j k l}^{*}\left(x, t-t^{\prime}\right) \mathrm{d} t^{\prime}=C_{i j k l}(x, t)$. See Section "The physics of time invariant..." vII The Class VII PRs are path and strain independent and only functions of time.

lastic PR time functions are based on specific loading or strain histories and, consequently, are limited to those conditions and are non-exportable to other loadings, since viscoelastic PRs are path dependent and hence non-unique as seen in Figure 1. In this figure, distinct PRs are displayed that were obtained from experimental strain data under different loading conditions on identical material specimen. Similar multiple PR time functions were reported in [113].

The Class VII PR definition avoids loading path dependence problems, but still leads to double convolution integrals in the time space that are extremely CPU intensive.

A recent paper [114], while not directly related to elastic PRs, analyzes the effects of geometry on changes in stress directions and demonstrates how usual 


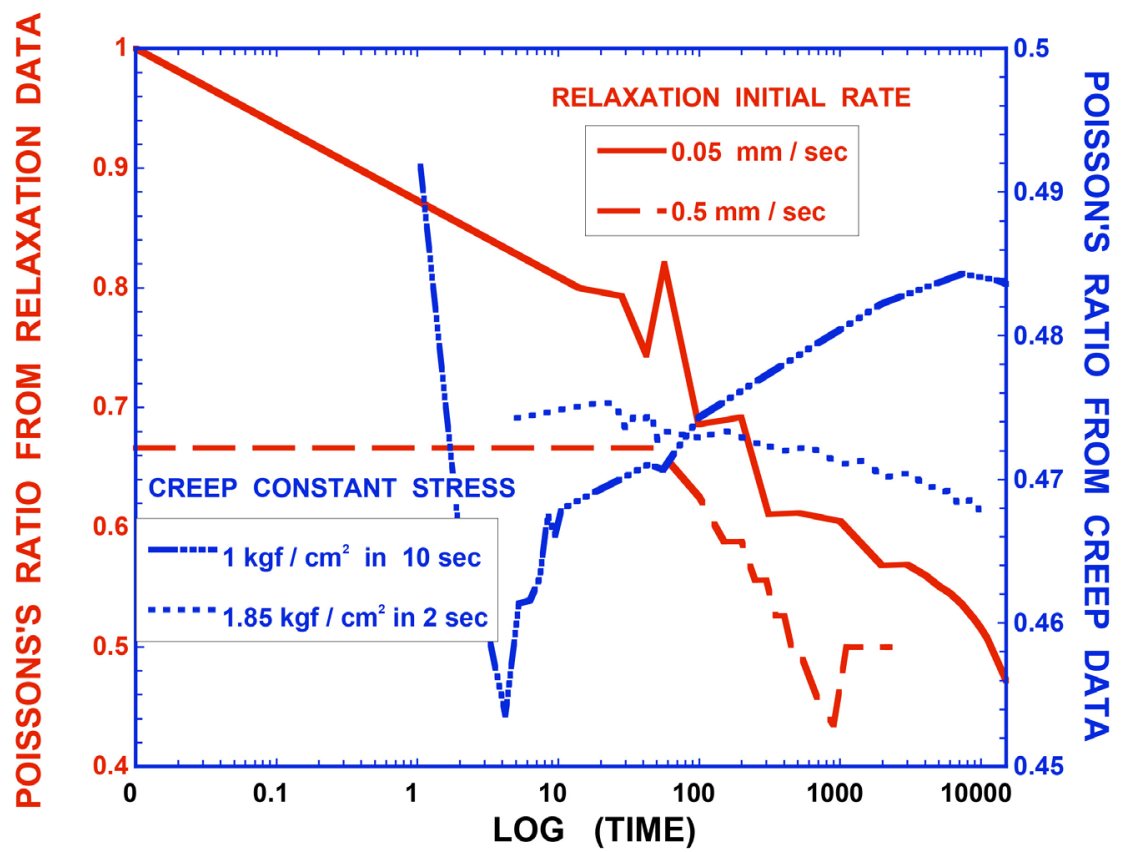

Figure 1. Case I Poisson's ratios based on creep and relaxation experiments [102].

tension strains can reverse direction, a phenomenon which the authors denote by "negative stiffness."

In [94] an analysis is presented on how to interpret loading over-and undershoots when extracting viscoelastic material properties from experimental data. An ancillary benefit is provided by demonstrating the importance of including loading phases in any analysis to determine viscoelastic material properties. Thus reinforcing the theoretical quasi-static and dynamic analysis results of [95] [96] that detail analytically the important effects of loading start-up phases on material characterizations.

However, as it is shown in [97]-[104] the entire issue of viscoelastic PRs can be by-passed by formulating 3-D analytical and experimental material characterizations in terms of 1-D loadings, and shear and bulk relaxation moduli or creep compliances. These characterizations are accomplished in either real time or Fourier or Laplace integral transform spaces and specifically include the associated loading cycles and totally steer clear of viscoelastic PR use. They are based on measurements of time, the 1-D stress and the 2-D strains and lead to universal viscoelastic material characterizations.

Comment 1. Aside from Poisson's great native genius, what made his namesake ratio universally accepted and most useful is that for all but seven decades of its two century existence the application were limited to elastic materials where for each of them it is a distinct constant. Unfortunately, nature was significantly less kind and far less magnanimous when it comes to viscoelasticity.

Detailed analyses and discussions of the above topics are presented in subsequent sections. 


\section{Linear Analysis}

\subsection{General Considerations}

Elastic and viscoelastic constitutive relations based on moduli and/or compliances are derived from fundamental principles, i.e. the thermodynamic laws pertaining to energy and entropy [17]. Thus, moduli and compliance parameters are thermodynamic derivatives in the same manner as for instance specific heats, etc.

In linear elastic materials moduli and compliances are time, path and stress independent and stress-strain relations involving PRs must be and are derived from these basic equations. Furthermore, in elasticity where PRs are constants and constitutive relations are algebraic, PRs are unique material properties and derivations are a straight forward matter.

In viscoelastic media where PRs are time, stress and stress history (path) dependent and where stress-strain relations involve time integrals multiple complications arise. For one, numerous PR definitions are possible and derivations may be carried out in either time space or in the integral transform space yielding in some instances mutually exclusive results and PR definitions. Secondly, since viscoelastic media dissipate energy loading histories take on prime importance and must be considered [95]-[104].

Figure 2 is a schematic representation of a loading path and moduli at various temperatures. The indicated times represent the following: $t_{0}$ is the time when relaxation begins, $t_{1}$ the time time when loading reaches the desired steadystate condition and $t_{\infty}$ the beginning time when the fully relaxed modulus starts. Clearly, the time at which the same loading path intercepts the modulus varies with temperature thereby eliciting distinct responses. Secondly, and equally importantly, if the buildup loading path from $0 \leq t \leq t_{1}$ is altered or if $t_{1}<t_{0}$, then the viscoelastic responses will change accordingly [95]-[104]. In [105] it is stated that transient rise times do not influence characterization if data is collected after ten times the rise time $t_{1}$. However, such an approximation ignores the relation of the rise time to the time $t_{0}$ when relaxation and/or creep begin-see Figure 2 and is not born out by either the subsequent exact analytical simulations of [95] and [96] or the experimental results presented in [97]-[104]. The rise time phenomenon is analyzed in detail in a later section.

The $P R$ analyses and formulations are generalized to non-homogeneous linear viscoelastic materials, however the main focus is on PR time and process dependence. The results apply equally to homogeneous viscoelastic media and PRs are path dependent for either homogeneous or non-homogeneous viscoelastic media.

Table 1 and Table 2 depict the various PR definitions of the six listed categories (classes) in real time space or in integral transform space. All are implicitly path (stress history) dependent, while Classes II and VI are implicitly so being respectively functions of constant strain and of constant stress loadings. Class IV involves a nonlinear definition involving natural strains and thus inapplicable to 
linear viscoelasticity.

Thus, while some of the viscoelastic PR models are defined in terms of consistent mathematical transient loading models, which are physically unattainable, they fail to translate into realistic and observable physical entities.

It will be shown that PRs, although extremely important in elastic material characterizations because there they are constants for each material, are not fundamental viscoelastic quantities in the same sense as relaxation moduli and creep compliances. Instead, they are multiply defined process dependent quantities as detailed in Table 1 and Table 2 and not interchangeable between divers loading conditions. Consequently, viscoelastic material characterizations are best expressed in terms of moduli or compliances [87] [88] [89] [90].

\subsection{Elastic PRs and Constitutive Relations}

Since elastic conditions form the ICs of any viscoelastic formulation and to properly establish EVCPs, it is necessary to first review pertinent elastic PR developments. Consider a Cartesian system with coordinates $x_{i}$, with $i=1,2,3$ and where $x=\left\{x_{1}, x_{2}, x_{3}\right\}$. The Einstein tensor notation is applied with pairs of repeated indices indicating summation and underlined ones signifying no summations. Isotropic and anisotropic elastic material behavior may be characterized in terms Young's moduli $E^{E}$, moduli $E_{i j k l}^{E}$, shear $G^{E}$ and bulk $K^{E}$ moduli, compliances $C_{i j k l}^{E}$ and PRs $v_{i j}^{E}$ [117] [118] [119] [120] [121]. In linear homogeneous elasticity each of these parameters are constants uniquely associated with elastic material behavior. The PRs in question are the original legacy Class I ones

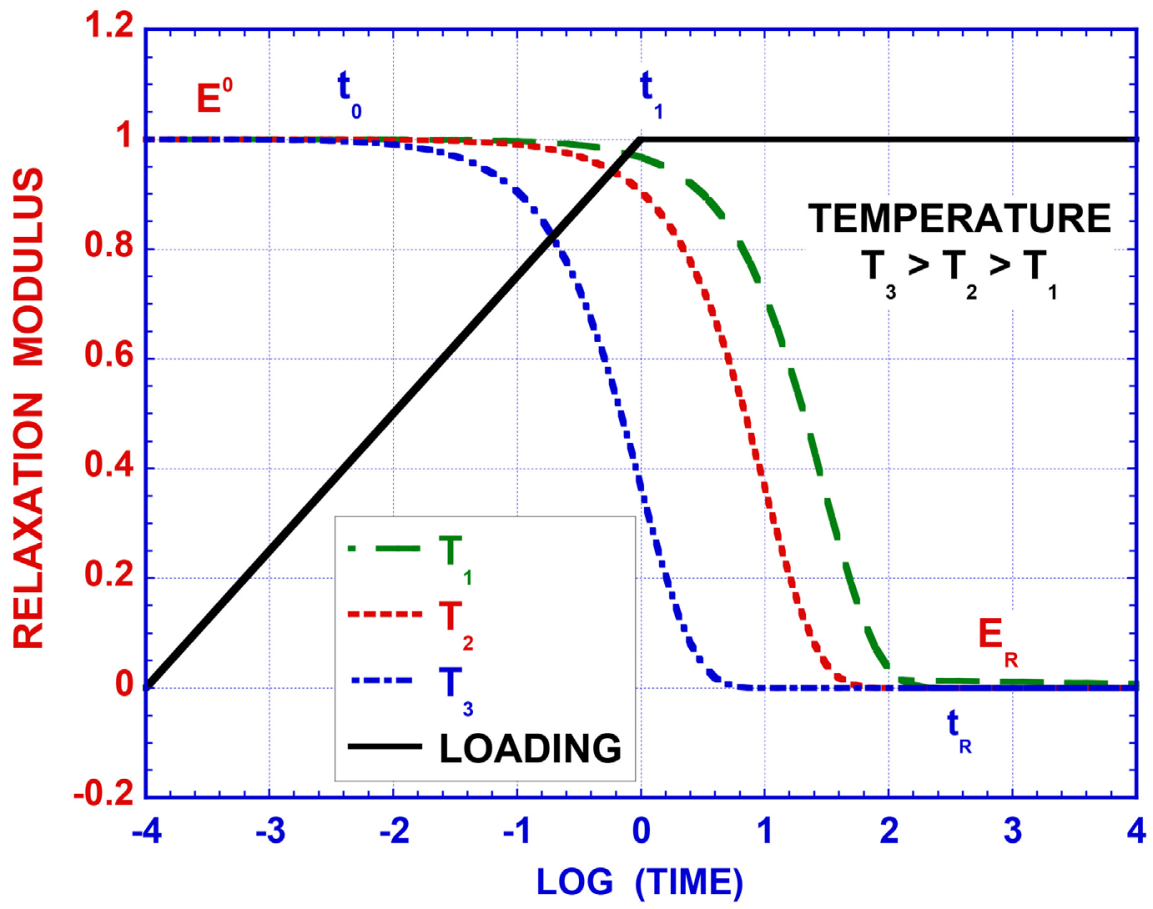

Figure 2. Schematic of typical viscoelastic moduli and loading pattern. 
Table 2. Viscoelastic Poisson's ratio definitions in time and Fourier transform (FT) [116] spaces.

\begin{tabular}{|c|c|c|}
\hline Class & Time Space PR $\left(\boldsymbol{v}_{\boldsymbol{i j}}(\boldsymbol{x}, \boldsymbol{t}), \boldsymbol{i} \neq \boldsymbol{j}, \boldsymbol{i} \neq \boldsymbol{j}\right)$ & FT Space PR $\left(\overline{\overline{\boldsymbol{v}}}_{i j}(\boldsymbol{x}, \boldsymbol{\omega}), \boldsymbol{i} \neq \boldsymbol{j}, \boldsymbol{i} \neq \boldsymbol{j}\right)$ \\
\hline I & $v_{i j}^{I}\left[x, t, \sigma_{\underline{k k}}(x, t)\right]=v_{i j}(x, t) \underline{\underline{\operatorname{def}}}-\frac{\epsilon_{i j}(x, t)}{\epsilon_{i i}(x, t)}$ & $\overline{\bar{v}}_{i j}^{I}(x, \omega)=-\int_{-\infty}^{\infty} \frac{\epsilon_{i j}(x, t)}{\epsilon_{i i}(x, t)} \exp (\imath \omega t) \mathrm{d} t$ \\
\hline II & $v_{i j}^{I I}(x, t) \underline{\underline{\operatorname{def}}}-\frac{\epsilon_{i j}(x, t)}{\epsilon_{i i}(x)}$ & $\overline{\bar{V}}_{i j}^{I I}(x, \omega)=-\frac{\overline{\bar{\epsilon}}_{\underline{i j}}(x, \omega)}{\epsilon_{i i}(x)}$ \\
\hline \multirow{4}{*}{ III } & $\epsilon_{i i}(x), t \in[0, \infty]$ mathematically OK, & $\begin{array}{l}\text { but step function physically } \\
\text { unachievable }\end{array}$ \\
\hline & $v_{i j}^{I I I}(x, t)=-\int_{-\infty}^{\infty} \frac{\overline{\bar{\epsilon}}_{i j}(x, \omega)}{\overline{\overline{\bar{t}_{i j}}}(x, \omega)} \mathrm{e}^{(-\imath \omega t)} \mathrm{d} \omega$ & $\overline{\bar{v}}_{i j}^{I I I}(x, \omega) \underline{\underline{\mathrm{def}}}-\frac{\overline{\bar{\epsilon}}_{i j}(x, \omega)}{\overline{\overline{\epsilon_{i i}}}(x, \omega)}$ \\
\hline & alternate equivalent definition (67) - (74): & see Eqs. (36) for lack of proper \\
\hline & $\epsilon_{\underline{i j}}(x, t) \underline{\underline{\mathrm{def}}}-\int_{-\infty} v_{i j}^{I I I}\left(x, t-t^{\prime}\right) \epsilon_{i i}\left(x, t^{\prime}\right) \mathrm{d} t^{\prime}$ & $\begin{array}{c}\text { equivalent physical relation in } t \\
\text { space }\end{array}$ \\
\hline \multirow[t]{3}{*}{ IV } & $v_{i j}^{I V}(x, t) \operatorname{def}-\frac{\log \left[1+\epsilon_{i j}(x, t)\right]}{\Gamma}$ & $\overline{\bar{V}}_{i j}^{V}(x, \omega)=-\int^{\infty} \frac{\log \left[1+\epsilon_{\underline{j i j}}(x, t)\right]}{{ }^{\prime}} \mathrm{e}^{(\omega t)} \mathrm{d} t$ \\
\hline & $\log \left[1+\epsilon_{i i}(x, t)\right]$ & $\int_{-\infty} \log \left[1+\epsilon_{i i}(x, t)\right]$ \\
\hline & $\partial v^{V}(x, t) \quad \frac{\partial \epsilon_{i j}(x, t)}{\partial t}$ & $\infty \frac{\partial \epsilon_{i j}(x, t)}{2}$ \\
\hline $\mathrm{V}$ & $\frac{\partial v_{i j}^{i j}(x, t)}{\partial t} \underline{\underline{\operatorname{def}}}-\frac{\partial t}{\underline{\partial \epsilon_{i i}(x, t)}}$ & $\overline{\bar{v}}_{i j}^{V}(x, \omega)=-\int_{-\infty}^{\infty} \frac{\partial t}{\frac{\partial \epsilon_{i i}(x, t)}{t(t \omega t)}} \frac{\mathrm{d} t}{l \omega}$ \\
\hline & $\partial t$ & $\partial t$ \\
\hline \multirow{5}{*}{ VI } & $v^{V I}(x, t) \operatorname{def}-\frac{C_{i j}(x, t)}{\underline{I}}$ & $-\int^{\infty} \underline{C}^{C_{i j i}(x, t)} \mathrm{e}^{(t o t)} \mathrm{d} t$ \\
\hline & $V_{1 j}(x, t) \underline{\underline{\mathrm{deI}}}-\overline{C_{1111}(x, t)}$ & $-\int_{-\infty} \overline{C_{1111}(x, t)}$ e $\mathrm{d} t$ \\
\hline & for $\sigma_{11}(x)$ only, all other $\sigma_{i j}=0$ & same physical restriction as Class II \\
\hline & for $t \in[-\infty, \infty]$ or $t \in[0, \infty]$ & $\begin{array}{c}\text { plus } \sigma_{11} \begin{array}{c}\text { must be time independent } \\
\text { over }\end{array}\end{array}$ \\
\hline & & the entire time interval $-\infty \leq t \leq \infty$ \\
\hline \multirow{3}{*}{ VII } & $C_{1}(x, t)=-\int v_{1 .}^{V I I}\left(x, t-t^{\prime}\right) C\left(x, t^{\prime}\right) \mathrm{d} t^{\prime}$ & $\overline{\bar{v}}_{1 j}^{V I I}(x, \omega) \operatorname{def}-\underline{\overline{\bar{C}}_{11 \underline{j i}}(x, \omega)}$ \\
\hline & 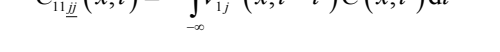 & $\overline{\bar{C}}(x, \omega)$ \\
\hline & this Class should be the PR of choice as it & $\begin{array}{c}\text { is path independent and hence } \\
\text { universal }\end{array}$ \\
\hline
\end{tabular}

defined $^{1}$ in [2] and [3] as the ratio of two isothermal orthogonal strains ${ }^{2}$ or

$$
v_{i j}=v_{i j}^{I} \underline{\underline{\operatorname{def}}}-\frac{\epsilon_{i j}(x, t)}{\epsilon_{i \underline{i}}(x, t)} \quad \text { with } \quad i, j=1,2,3 \text { and } i \neq j
$$

The anisotropic homogenous elastic constitutive relations read

$$
\epsilon_{i j}^{E}(x, t)=C_{i j k l}^{E} \sigma_{k l}^{E}(x, t)+\overbrace{\alpha_{i j} \vartheta(x, t)}^{\substack{\text { thermal } \\ \text { strain }}} \text { and } \sigma_{i j}^{E}(x, t)=E_{i j k l}^{E}\left[\epsilon_{k l}^{E}(x, t)-\alpha_{\underline{i j}} \vartheta(x, t)\right](2)
$$

with $\vartheta(x, t)=T(x, t)-T_{0}(x, t)$ where $T_{0}$ is a reference temperature when the thermal trains are zero. In the isothermal cases $\vartheta(x, t)=0$.

In particular, for a 1-D loading $\sigma_{11} \neq 0$ and all other $\sigma_{i j}=0$

${ }^{1}$ For definitions of five other PR Classes or Categories see [88], [90] and Table 1 and Table 2.

${ }^{2}$ For example in a 1-D stress field with $\sigma_{11} \neq 0$, the strain $\epsilon_{11}$ is the one in the loaded direction and $\epsilon_{22}$ is the transverse strain component. 


$$
v_{1 j}^{E}=-\frac{C_{i j 11}^{E}}{C_{1111}^{E}}=-\frac{C_{i j 11}^{E}}{C^{E}} \text { with } j=2,3 \text { for } \sigma_{11}(x, t) \neq 0, \text { all other } \sigma_{i j}=0
$$

with the $C_{i j k l}^{E}$ equal to constants that are specific to each elastic medium. Generally $C_{j i 11}^{E} \neq C^{E}$ except when $v_{i j}^{E}=-1$.

However, for non-homogeneous elastic materials the usually constant 1-D PRs become spatial functions with values depending still on distinct material properties but also additionally on the position $x=\left\{x_{1}, x_{2}, x_{3}\right\}$ within the elastic body, such that Eqs. (3) turn into [85]

$$
v_{1 j}^{E}(x)=-\frac{C_{i j 11}^{E}(x)}{C_{1111}^{E}(x)} \text { with } j=2,3 \text { for } \sigma_{11}(x) \neq 0 \text {, all other } \sigma_{i j}=0
$$

For linear homogeneous elastic media, it can be readily proven that $-1 \leq v_{i j}^{E} \leq 0.5$ see [117] to [121]. However, in non homogeneous elastic bodies no such restrictions apply [85]. Nor is the PR an absolute constant for each isotropic isothermal non-homogeneous elastic medium, but rather varies spatially in a manner similar to the corresponding moduli and compliances [85].

Table 3 and Figure 3 display the various moduli for the isotropic isothermal homogenous elastic media based on Hooke's law. These are seen to vary from an incompressible material at $v^{E}=0.5$ to a rigid one in shear with only tension or compressive strains at a PR of 1 . Parenthetically, one must add that these limits do not apply in linear viscoelasticity, except at $t=0$ for those viscoelastic media that have elastic initial conditions.

It can be further demonstrated that for linear elastic media, the following hold true

$$
\begin{gathered}
E^{E}(x)=\frac{3 G^{E}(x)}{1+G^{E}(x) / K^{E}(x)} \\
K^{E}(x)=\frac{E^{E}(x)}{1-2 v^{E}(x)} \text { and } G^{E}(x)=\frac{E^{E}(x)}{2\left[1+v^{E}(x)\right]}
\end{gathered}
$$

and similar relations for the Lamé parameters (constants)

$$
\lambda^{E}(x)=\frac{v^{E}(x) E^{E}(x)}{\left[1+v^{E}(x)\right]\left[1-2 v^{E}(x)\right]} \text { and } \mu^{E}(x)=G^{E}(x)
$$

Alternate elastic constitutive relations are obtained by inverting the set (2) which yields

$$
\begin{aligned}
\sigma_{11}^{E}(x, t)= & \frac{E^{E}(x)}{\left[1+v^{E}(x)\right]\left[1-2 v^{E}(x)\right]}\left(\left[1-v^{E}(x)\right] \epsilon_{11}^{E}(x, t)\right. \\
& \left.+v^{E}(x)\left[\epsilon_{22}^{E}(x, t)+\epsilon_{33}^{E}(x)\right]\right)+\frac{E^{E}(x) \alpha \vartheta(x, t)}{1-2 v^{E}(x)} \\
\text { with }-1 \leq & v^{E}<\frac{1}{2} \\
& \sigma_{12}^{E}(x, t)=2 G^{E}(x) \epsilon_{12}^{E}(x, t) \text { etc. }
\end{aligned}
$$


Table 3. Relations between isotropic linear elastic moduli and Poisson's ratios.

\begin{tabular}{cccc}
\hline$P R^{E}$ & $K^{E}$ & $E^{E}$ & $G^{E}$ \\
\hline 0.5 & $\infty$ & $3 G^{E}$ & $E^{E} / 3$ \\
0 & $E^{E}=2 G^{E}$ & $2 G^{E}=K^{E}$ & $K^{E} / 2=E^{E} / 2$ \\
-0.5 & $E^{E} / 2=G^{E} / 2$ & $2 K^{E}=G^{E}$ & $2 K^{E}=E^{E}$ \\
-1 & $E^{E} / 3$ & $3 K^{E}$ & $\infty$ \\
\hline
\end{tabular}

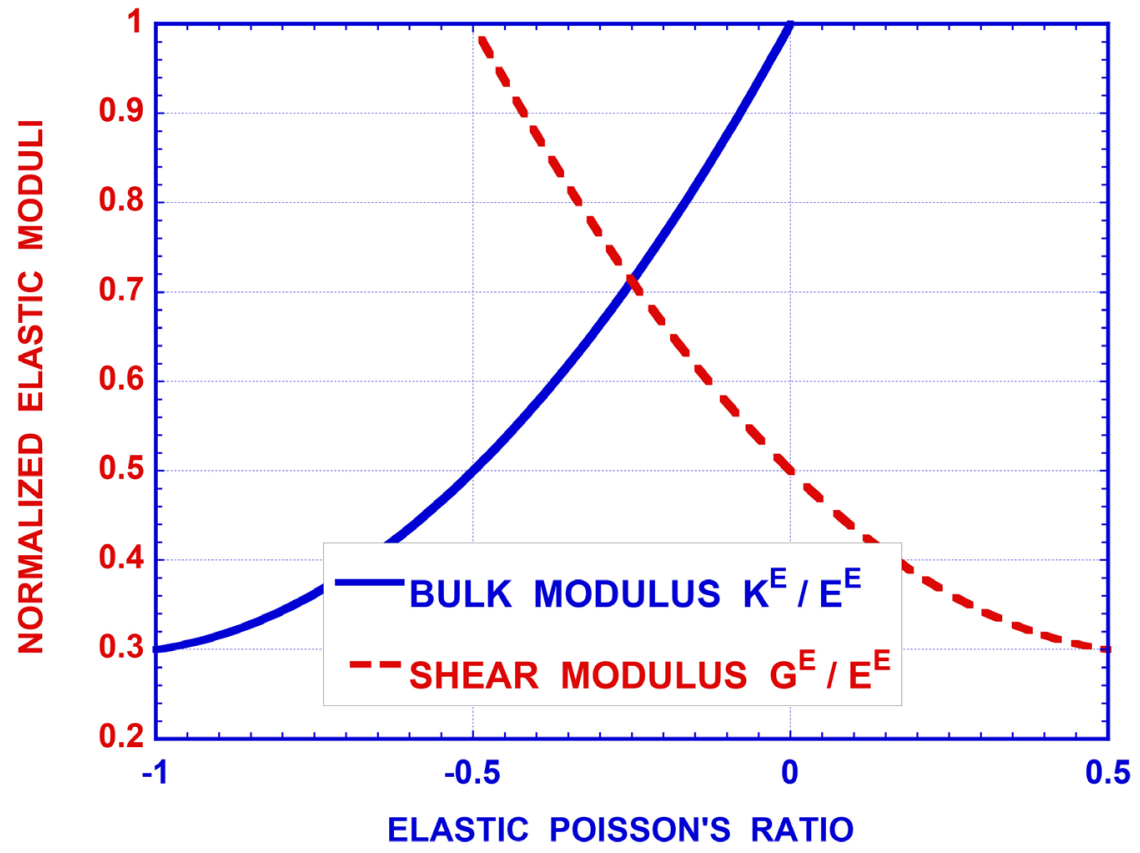

Figure 3. Elastic moduli and Poisson's ratio.

\subsection{3-D Hooke's Law and Poisson Ratio Induced Nonlinearities}

In linear 1-D elasticity, the isotropic isothermal Hooke's law simplifies to

$$
\begin{aligned}
\epsilon_{11}^{E}(x, t) & =\frac{\sigma_{11}^{E}(x, t)}{E^{E}(x)}=C^{E}(x) \sigma_{11}^{E}(x, t) \\
\epsilon_{\underline{j j}}^{E}(x, t) & =-\frac{v^{E}(x) \sigma_{11}^{E}(x, t)}{E^{E}(x)} \\
& =-v^{E}(x) C^{E}(x) \sigma_{11}^{E}(x, t) \\
& =C_{1122}^{E}(x) \sigma_{11}^{E}(x, t) \quad j \neq 1
\end{aligned}
$$

where $C^{E}$ is the elastic compliance.

Furthermore, in conservative linear systems Maxwell's reciprocal theorem [117] [118] [119] applies and the loading order is immaterial for linear elastic media. Therefore, in 2 or 3-D linear isotropic elasticity the strain $\epsilon_{11}^{E}$ is determined by assuming or prescribing that $\sigma_{11}^{E}$ is applied first and then followed by the other two normal stresses. As derived in [118], geometric considerations reveal the inherent PR nonlinearities based on a linear elastic Hooke's law 


$$
\begin{aligned}
& \epsilon_{11}^{E}(x, t)=\underbrace{\frac{\sigma_{11}^{E}(x, t)}{E^{E}(x)}}_{\begin{array}{c}
=\text { longitudinal } \\
\text { linear term }
\end{array}}-\underbrace{\frac{v^{E}(x)\left[\sigma_{22}^{E}(x, t)+\sigma_{33}^{E}(x, t)\right]}{E^{E}(x)}}_{\begin{array}{c}
\text { = transverse stress contributions, } \\
\text { linear term }
\end{array}} \underbrace{\left[+\frac{\sigma_{11}^{E}(x, t)}{E^{E}(x)}\right]}_{\begin{array}{c}
\text { = longitudinal stress } \\
\text { influence } \\
\text { nonlinear term }
\end{array}} \\
& \approx \frac{\sigma_{11}^{E}(x, t)}{E^{E}(x)}-\frac{v^{E}(x)\left[\sigma_{22}^{E}(x, t)+\sigma_{33}^{E}(x, t)\right]}{E^{E}(x)} \text { for } \frac{\sigma_{11}^{E}(x, t)}{E^{E}(x)} \ll 4
\end{aligned}
$$

Taking the FT of these linearized isotropic elastic constitutive relations yields

$$
\begin{aligned}
\overline{\bar{\epsilon}}_{11}(x, \omega) & \approx \frac{\overline{\bar{\sigma}}_{11}^{E}(x, \omega)}{E^{E}(x)}-\frac{v^{E}(x)\left[\overline{\bar{\sigma}}_{22}^{E}(x, \omega)+\overline{\bar{\sigma}}_{33}^{E}(x, \omega)\right]}{E^{E}(x)} \text { for } \frac{\sigma_{11}^{E}(x, t)}{E_{\text {linearization term }}^{E}(x)} \ll \\
& \approx C_{111}^{E} \overline{\bar{\sigma}}_{11}^{E}(x, \omega)+C_{1122}^{E}\left[\overline{\bar{\sigma}}_{22}^{E}(x, \omega)+\overline{\bar{\sigma}}_{33}^{E}(x, \omega)\right]
\end{aligned}
$$

Comment 2. It should be noted that in most current viscoelastic materials, such as high polymers, rubbers, etc., the inequality (linearization term) in (11) and (12) is often violated because of low $E^{E}$ and $E(t)$ values comparable to and sometimes smaller than $\sigma_{11}^{E}$. The inclusion of this approximation is crucial to linear PR elasticity and the admissibility of the EVCP based on PRs. On the other hand, constitutive relations in terms of moduli andlor compliances are formulated from fundamental deviatoric and volumetric laws deformation without the need to invoke the linearization assumption.

The net effect of this linearization term is that linear elastic materials possess linear modulus/compliance constitutive relations, but potentially nonlinear ones when PR formulations are included.

However, the above linearization must be enforced since the EVCP depends on integral transforms, which can only be applied to linear systems. Additionally and separately, in order to formulate the EVCP in terms of PRs it is necessary to find a term by term and parameter by parameter viscoelastic match in the FT space to the elastic transformed relations (12), with a corresponding valid and plausible viscoelastic set of constitutive relations in the time space.

Alternately, the troublesome nonlinearity can be totally avoided if Hooke's law is derived from fundamental deviatoric (shape change) and volumetric (volume changes) constitutive relations and by applying the three normal stresses simultaneously, to whit

$$
\begin{gathered}
\text { deviatoric } \Rightarrow \epsilon_{11}^{E}-\frac{\epsilon_{i i}^{E}}{3}=\frac{1}{2 G^{E}}\left(\sigma_{11}^{E}-\frac{\sigma_{i i}^{E}}{3}\right) \\
\text { volumetric } \Rightarrow \epsilon_{i i}^{E}=\frac{\sigma_{i i}^{E}}{K^{E}}
\end{gathered}
$$

Substitution of (14) into (13) yields

$$
\epsilon_{11}^{E}=\underbrace{\frac{1+G^{E} / K^{E}}{3 G^{E}}}_{=1 / E_{111}^{E}=C_{1111}^{E}} \sigma_{11}^{E}+\underbrace{\left(\frac{1}{3 K^{E}}-\frac{1}{6 G^{E}}\right)}_{=C_{1122}^{E}}\left(\sigma_{22}^{E}+\sigma_{33}^{E}\right)
$$


and one equation for each of the other two normal strains. Eq. (15) and its other two cousins are unambiguously linear and do not suffer from the nonlinearities of the PR relations (11). Furthermore in the latter formulation all three normal loads are applied simultaneously which is of prime importance in dissipate systems such as viscoelastic or plastic media.

\subsection{Viscoelastic PRs and Constitutive Relations}

Whereas in isotropic isothermal homogeneous linear elasticity the PR values are constants and material specific, the circumstances are far different for their viscoelastic cousins under the same specifications.

Consider the general linear anisotropic isothermal viscoelastic relations [29][75], which are the counterparts to Eqs. (2)

$$
\begin{aligned}
\epsilon_{i j}(x, t)= & \int_{-\infty}^{t} C_{i j k l}\left[x, t, t^{\prime}, T\left(x, t^{\prime}\right)\right] \frac{\partial \sigma_{k l}\left(x, t^{\prime}\right)}{\partial t^{\prime}} \mathrm{d} t^{\prime} \\
& +\int_{-\infty}^{t} C_{i j}^{T}\left[x, t, t^{\prime}, T\left(x, t^{\prime}\right)\right] \frac{\partial\left[\alpha \vartheta\left(x, t^{\prime}\right)\right]}{\partial t^{\prime}} \mathrm{d} t^{\prime} \\
\sigma_{i j}(x, t)= & \int_{-\infty}^{t} E_{i j k l}\left[x, t, t^{\prime}, T\left(x, t^{\prime}\right)\right] \frac{\partial \varepsilon_{k l}\left(x, t^{\prime}\right)}{\partial t^{\prime}} \mathrm{d} t^{\prime} \\
& -\int_{-\infty}^{t} E_{i j}^{T}\left[x, t, t, T\left(x, t^{\prime}\right)\right] \frac{\partial\left[\alpha \vartheta\left(x, t^{\prime}\right)\right]}{\partial t^{\prime}} \mathrm{d} t^{\prime}
\end{aligned}
$$

Consequently, the Class I viscoelastic PR for an isothermal process when $T=0$ becomes

$$
\begin{aligned}
v_{i j}(x, t) & =v_{i j}^{I}(x, t)=-\frac{\int_{-\infty}^{t} C_{\underline{j i k l}}\left(x, t-t^{\prime}\right) \frac{\partial \sigma_{k l}\left(x, t^{\prime}\right)}{\partial t^{\prime}} \mathrm{d} t^{\prime}}{\int_{-\infty}^{t} C_{\underline{i i m n}}\left(x, t-t^{\prime}\right) \frac{\partial \sigma_{m n}\left(x, t^{\prime}\right)}{\partial t^{\prime}} \mathrm{d} t^{\prime}} \\
& =-\frac{\int_{-\infty}^{t} C_{\underline{j} k k l}^{*}\left(x, t-t^{\prime}\right) \sigma_{k l}\left(x, t^{\prime}\right) \mathrm{d} t^{\prime}}{\int_{-\infty}^{t} C_{\underline{i i m n}}^{*}\left(x, t-t^{\prime}\right) \sigma_{m n}\left(x, t^{\prime}\right) \mathrm{d} t^{\prime}} \quad i \neq j \quad i, j=1,2,3
\end{aligned}
$$

Note that even in the simplest 1-D isotropic problem when only one stress derivative is present and if it is equal to a constant value ${ }^{3}$ for $0 \leq t \leq \infty$ and if the material is homogeneous, then the viscoelastic PR is still a time function, i.e. $v_{12}(t)=v_{13}(t)$. This is due to the fact that for an isotropic homogeneous material

$$
\begin{gathered}
C_{1111}(t) \neq C_{1122}(t)=C_{1133}(t) \quad \text { and } \quad C_{11 \underline{m m}}(t)=0 \quad m=4,5,6 \\
C_{1111}(t)=C_{2222}(t)=C_{3333}(t) \text { and } \quad \overline{\bar{C}}_{\underline{m m m m}}(\omega)=\frac{1}{2(\imath \omega)^{2} \overline{\bar{G}}(\omega)} \quad m=4,5,6
\end{gathered}
$$

${ }^{3}$ See the Section "The physics of time invariant stresses, strains and their derivatives" on the physical aspects and implications of time invariant stresses, strains or their time derivatives, particularly during actual loading phases. 


$$
C_{\underline{k k l l}}(t)=C_{\underline{l k k k}}(t) \quad k, l=1,2, \cdots, 6
$$

Comment 3. In other instances when the stress or strain derivatives are time functions, they are "trapped" in the integrals and the PRs become time, stress and stress history dependent, i.e. process dependent [87] [88] [89] [90]. Therefore, the viscoelastic PRs are no longer pure unique material property characterizations in the sense of moduli and compliances, and are, therefore, unsuitable as material descriptors. This applies to Classes I through VI. ClassVI (1-D constant stress) and Class II (1-D constant strain) are special limited cases, and in reality none of the PRs are exportable from one loading condition or from one Class to another including time independent loadings.

In the very special case when $C_{1111}(t)=C_{1122}(t)=C_{1133}(t)$, the shear modulus $G(t)$ is related to the bulk modulus $K(t)$ through a constant multiplier with the requirement that their relaxation times are equal [86]. Real materials generally exhibit $K(t) \gg G(t)$ except for the auxetic ones where the inequality reversed. In either instance Eq. (18a) is satisfied.

The relaxation moduli and creep compliances are as a matter of convenience generally represented by Prony series [122] of the type

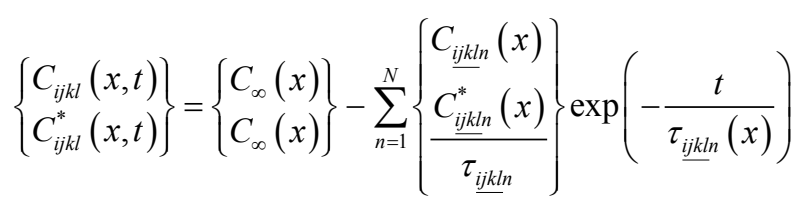

with similar expressions for $E_{i j k l}(x, t)$ and $E_{i j k l}^{*}(x, t)$.

Fourier transform (FT) [15] are introduced in preference to Laplace transforms (LT) as they are more inclusive and extends from $-\infty$, where all state variables are at rest, to the current time $t$ at which the solution is sought. Consequently, in general analyses such as those presented here ICs at $t=0$ need not be included and need not be specified until solutions of particular problems are undertaken. FTs were first introduced to viscoelasticity in order to derive the integral EVCP in Ref. [16] and are defined here as

$$
\mathfrak{F}\{f(x, t)\}=\overline{\bar{f}}(x, \omega)=\int_{\infty}^{\infty} f(x, t) \exp (\iota \omega t) \mathrm{d} t
$$

The FTs are equivalent to the two sided LTs as stated by the Rodrigues formula and provided all integrals exist these transforms are related to each other by [116] [123]

$$
\underbrace{\overline{\bar{f}}(x, \omega)}_{=F T}=\left.\underbrace{\bar{f}(x, p)}_{=L T}\right|_{p=-\iota \omega}=\left.\int_{-\infty}^{0} f(x, t) \exp (-p t) \mathrm{d} t\right|_{p=-l \omega}+\left.\int_{0}^{\infty} f(x, t) \exp (-p t) \mathrm{d} t\right|_{p=-\iota \omega}
$$

Application of FTs converts the convolution integral isothermal constitutive relations (16) to

$$
\overline{\bar{\epsilon}}_{i j}(x, \omega)=\imath \omega \overline{\bar{C}}_{i j k l}(x, \omega) \overline{\bar{\sigma}}_{k l}(x, \omega)
$$




$$
\overline{\bar{\sigma}}_{i j}(x, \omega)=\imath \omega \overline{\bar{E}}_{i j k l}(x, \omega) \overline{\bar{\epsilon}}_{k l}(x, \omega)
$$

Class VI PR expressions are derived in [90], while all other Categories have defined expressions as seen in [87]. The Class VII PRs have their genesis in a definition starting in the FT space, based on a form of the constitutive relations that can be interpreted as supporting an EVCP. However, they are devoid of physical meaning and the stress-strain relations do not transform into a form in time space that can be derived independently from thermodynamic first principles. They represent a group of material property time functions independent of stress and strain histories. Nevertheless, their utility is far less than the relaxation moduli and creep compliances that define them because they are specific to a limited 1-D loading condition from which one cannot generalize the PR functions.

The Elastic-Viscoelastic Correspondence Principle or Analogy (EVCP) ${ }^{4}$

The EVCP, when applicable, is a powerful protocol for solving linear viscoelastic problems based on similarities between integral transform (IT) elastic and viscoelastic formulations and solutions. EVCP comes in two varieties: separation of variables [14] and the considerably less restrictive one based on integral transforms [16]. The IT EVCP consists of substituting complex viscoelastic moduli/compliances for their elastic equivalences in Laplace (LT) or Fourier (FT) transforms elastic solutions. This means that any analytical and some numerical elastic solutions are easily convertible to their equivalent viscoelastic solutions. In those instances where material properties do not conform to convolution integral constitutive relations, approximate EVCPs have been derived that are based on piecewise convolution integral representations [124] [125].

The EVCP can be formally stated as:

1) Given an elastic body with the FT of its general solution

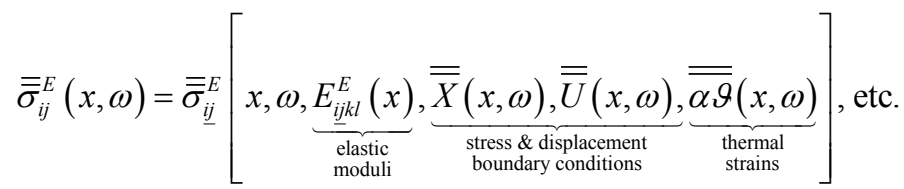

2) Then for viscoelastic convolution ${ }^{5}$ integral constitutive relations given by (16) and with identical boundary conditions, the viscoelastic solution is

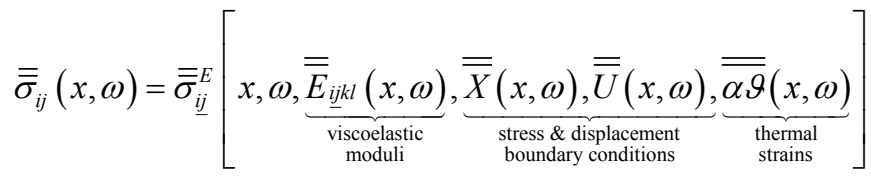

and

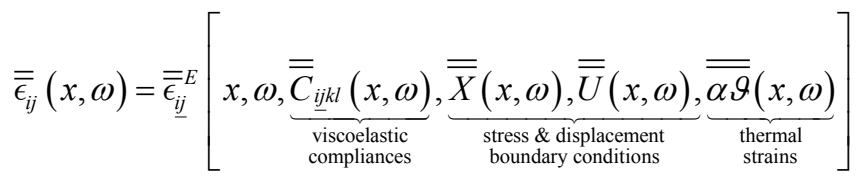

\footnotetext{
${ }^{4}$ Also see the section "The physics of time invariant stresses, etc."

${ }^{5}$ The EVCP is inapplicable if the material property are considered temperature dependent and if the
} temperature is a time function, i.e. $T=T(t)$ or $T(x, t)$. 
are universally valid provided the transforms and their inverses exist.

Comment 4. The EVCP protocol [16] for rigorously establishing Eqs. (23) to (25) is to first derive the pertinent viscoelastic governing relations and their generalized constitutive relations and solutions in the time space and then take their FT and compare these transforms to the FTs of the corresponding elastic solutions.

In order to properly highlight the difficulties of generating an EVCP involving viscoelastic PRs, it is necessary to first review the process by which Eqs. (2), the 3-D general Hooke's law with PRs is derived. Elasticity is a conservative system and its constitutive relations are algebraic. Whereas the viscoelastic system is a dissipative one with memory leading to integral differential constitutive relations in time, such as (16).

However, the linearization described in Section 3.3 must be enforced since the EVCP depends on integral transforms, which can only be applied to linear systems. Additionally and separately, in order to formulate the EVCP in terms of PRs it is necessary to find a term by term and parameter by parameter viscoelastic match in the FT space to the elastic transformed relations (12), with a corresponding valid and plausible viscoelastic set of constitutive relations in the time space.

In a non-conservative system such as a linear viscoelastic body, the luxury of choice of free sequencing of loads is no longer available, as different loading schedules result in distinct responses, i.e. Maxwel's reciprocal theorem is inapplicable. This is due to the material's memory, and consequent energy dissipation, which is expressed by integral type constitutive relations rather than the elastic algebraic ones. The viscoelastic ones also do not allow for the legitimate types of elastic algebraic approximations and manipulations of Eqs. (10) and (11), thus dictating the need for different approaches [90].

Since the correspondence principle has been proven inapplicable when PRs are involved [87] [88] [89], the usual elastic relations for shear and bulk moduli have no viscoelastic counterparts in terms of PRs, i.e.

$$
\begin{gathered}
\overline{\bar{G}} \neq \frac{\overline{\bar{E}}}{2\left(1+\overline{\overline{v^{I}}}\right)}, \quad \overline{\bar{K}} \neq \frac{\overline{\bar{E}}}{1-2 \overline{\overline{v^{I}}}}, \\
\overline{\bar{\sigma}}_{i j}(x, \omega) \neq \overline{\bar{\sigma}}_{i j}^{e}[x, \omega, \overline{\bar{E}}_{i j k l}(x, \omega), \overline{v^{I}}(x, \omega), \underbrace{\overline{\bar{X}}_{i}(x, \omega), \underbrace{\overline{\bar{U}}_{i}}_{\text {thermal strains }}(x, \omega), \overline{\overline{\alpha \vartheta}}(x, \omega)}_{\text {boundary conditions }}] \text { etc. }
\end{gathered}
$$

but without PRs, the expressions

$$
\overline{\bar{E}}(x, \omega)=\frac{3 \overline{\bar{G}}(x, \omega)}{1+\overline{\bar{G}}(x, \omega) / \overline{\bar{K}}(x, \omega)},
$$

and Eqs. (25) are valid.

However, if $\overline{\bar{v}}^{I I I}$ is substituted for $\overline{\bar{v}}^{I}$ in (26) then the resulting relations 


$$
\begin{gathered}
\overline{\bar{G}}(x, \omega)=\frac{\overline{\bar{E}}(x, \omega)}{2\left[1+\overline{\overline{v^{I I I}}}(x, \omega)\right]}, \quad \overline{\bar{K}}(x, \omega)=\frac{\overline{\bar{E}}(x, \omega)}{1-2 \overline{\overline{v^{I I I}}}(x, \omega)}, \\
\overline{\bar{\sigma}}_{i j}(x, \omega)=\overline{\bar{\sigma}}_{i j}^{e}[x, \omega, \overline{\bar{E}}_{i j k l}(x, \omega), \overline{\overline{v^{I I I}}}(x, \omega), \underbrace{\overline{\bar{X}}_{i}(x, \omega), \underbrace{}_{\text {thermal strains }}], \quad \text { etc. }}_{\text {boundary conditions }}
\end{gathered}
$$

are valid subject to the limitations of the non-uniqueness of the viscoelastic PRs due to their stress and path (stress history) dependence. Difficulties with generating a proper EVCP in terms of PRs stem from the following concepts, which will be established below in detail, see Eqs. (32).

1) The time and separate stress history dependence of the viscoelastic PRs, i.e. experimental conditions do not necessarily reflect in-service situations.

2) The inherent assemblage of individual material contributors in the elastic constitutive relations when PRs are involved calling for a triple product of material property parameters and stress tensors.

3) The viscoelastic PR triple product while obligatory in the Fourier transform space for establishing an EVCP has limited physical counterparts in the real time space nor any relation to the Boltzmann superposition principle [6], except for one model (32b).

In terms of moduli/compliances the 3-D Hooke's law and the corresponding linear viscoelastic constitutive relations are both derivable from first principles [17] to read for elastic media

$$
\epsilon_{i j}^{E}(x, t)=C_{\underline{i j k l}}^{E}(x)\left[\sigma_{k l}^{E}(x, t)+\delta_{\underline{i j}} \alpha \vartheta(x, t)\right] .
$$

However, when the isotropic Hooke's law is assembled with elastic PRs, it is in an almost ad hoc manner of one direction at a time to yield ${ }^{6}[120]$

$$
\epsilon_{i j}^{E}(x, t)=\underbrace{\frac{1}{E^{E}(x)}}_{=C^{E}(x)}\left[\sigma_{\underline{i j}}^{E}(x, t)-v_{12}^{E}(x)\left(\sigma_{i \underline{i j}}^{E}(x, t)+\sigma_{\underline{k} \underline{k}}^{E}(x, t)\right)+\alpha \vartheta(x, t)\right] i \neq j \neq k .(30)
$$

Specifically, the pitfalls in producing a coherent EVCP arise from the triple product elastic combinations and their $\mathrm{FTs}^{7}$

$$
\frac{v_{12}^{E}(x) \sigma_{i i}^{E}(x, t)}{E^{E}(x)}=C^{E}(x) v_{12}^{E}(x) \sigma_{i i}^{E}(x, t) \Rightarrow C^{E}(x) v_{12}^{E}(x) \overline{\overline{\sigma_{i i}^{E}}}(x, \omega)
$$

that requires a viscoelastic triple FT product counterpart $\overline{\overline{C^{*}}}(x, \omega) \overline{\overline{v_{12}^{D}}}(x, \omega) \overline{\bar{\sigma}}_{i i}(x, \omega)$, which is generated in the integral transform space (FT or LT). However, in order to achieve any physical and mathematical credence this triple product needs to be generated from real time combinations to conform with its prescribed FT inversions. Therefore, starting with the need- ed ${ }^{6}$ cf. Eq. (11).

${ }^{7}$ This is but one of the two triple products in the constitutive relations. The other one involves $\sigma_{\underline{k}}$ and $\epsilon_{\underline{k k}}$ and it experiences an identical formulation in the constitutive relations for volume changes. 
triple PR FT product, the required isotropic isothermal constitutive relations in the time space must be the inverse FT

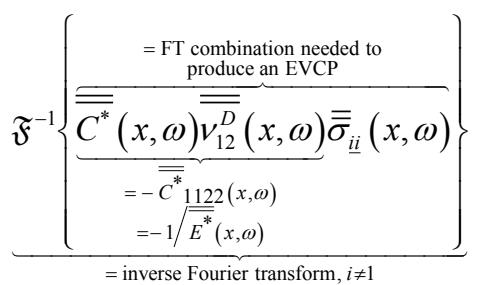

$$
\begin{aligned}
& \left\{\begin{array}{l}
\int_{-\infty}^{t} C^{*}\left(x, t-t^{\prime}\right)\left\{\int_{-\infty}^{t^{\prime}} v_{12}^{D}\left(x, t^{\prime}-s\right) \sigma_{\underline{i i}}(x, s) \mathrm{d} s\right\} \mathrm{d} t^{\prime} \\
\text { not OK in time space } \\
\int_{-\infty}^{t} \underbrace{\left\{\int_{-\infty}^{t^{\prime}} C^{*}\left(x, t^{\prime}\right)\right.}_{=-C_{1 i \underline{i}}^{*}\left(x, t^{\prime}\right), i \neq 1}
\end{array}\right. \\
& \text { OK in time space see (33) } \\
& =\left\{\int_{-\infty}^{t} v_{12}^{D}\left(x, t-t^{\prime}\right)\left\{\int_{-\infty}^{t^{\prime}} C^{*}\left(x, t^{\prime}-s\right) \sigma_{i i}(x, s) \mathrm{d} s\right\} \mathrm{d} t^{\prime}\right. \\
& \text { not } \mathrm{OK} \text { in time space } \\
& -\int_{-\infty}^{t} C_{11 i i}^{*}\left(x, t-t^{\prime}\right) \sigma_{i i}\left(x, t^{\prime}\right) \mathrm{d} t^{\prime} \\
& =-\int_{-\infty}^{t} C_{11 i i}\left(x, t-t^{\prime}\right) \frac{\partial \sigma_{i i}\left(x, t^{\prime}\right)}{\partial t^{\prime}} \mathrm{d} t^{\prime} \\
& \text { without PRs always universally true }
\end{aligned}
$$

where $v_{21}^{D}(x, t)=v_{12}^{D}(x, t)$ represent any class PR for which the FT triple product can be generated ${ }^{8}$. The above four expressions indicate how the constitutive relations must be formulated in the real time space so that the desired FT triple products can be realized. If the isotropic 1-D elastic protocol of Eqs. (10) is used in assembling equivalent viscoelastic PR constitutive relations, then the construction becomes

$$
\begin{aligned}
& \epsilon_{11}(x, t)=\int_{-\infty}^{t} C^{*}\left(x, t-t^{\prime}\right) \sigma_{11}\left(x, t^{\prime}\right) \mathrm{d} t^{\prime} \\
& \epsilon_{\underline{j}}(x, t)=-\int_{-\infty}^{t}\{\underbrace{\int_{-\infty}^{t^{\prime}} C^{*}\left(x, t^{\prime}-s\right) v_{12}^{D}(x, s) \mathrm{d} s}_{=C_{11 \underline{j \underline{j}}}^{*}\left(x, t t^{\prime}\right)}\} \sigma_{11}\left(x, t-t^{\prime}\right) \mathrm{d} t^{\prime} \quad j \neq 1
\end{aligned}
$$

It then follows from this inverse heuristic approach that

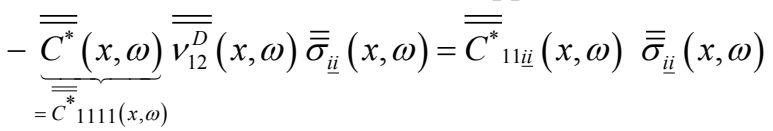

$$
\begin{aligned}
& =\underbrace{\overline{\bar{C}}_{11 i i}(x, \omega)}_{=\overline{\bar{C}}^{*} 11 i i(x, \omega)} \overline{\bar{\sigma}}_{i i}(x, \omega), i \neq 1
\end{aligned}
$$

${ }^{8}$ Eqs. (36) apply equally to linear homogeneous viscoelastic media. 
There are three Classes of PRs that can produce the form (34), namely Class II, III and VI. However, neither Class II or VI produce a physically valid expression in the real time space. Class III, as pointed out in earlier, has only one convolution form out three possible ones that produce a physical counterpart (32b).

The definition of the Class VI viscoelastic PR leads to

$$
\begin{gathered}
\overline{\overline{v_{12}^{V I}}}(x, \omega) \underline{\underline{\text { def }}}-\frac{\overline{\bar{C}}_{1122}(x, \omega) \sigma_{11}(x)}{\overline{\bar{C}}(x, \omega)) \sigma_{11}(x)}=-\overline{\bar{C}}_{1122}(x, \omega) \overline{\bar{E}}(x, \omega) \\
\text { with } \sigma_{11}(x, t)=\sigma_{11}(x) \text { only and all other } \sigma_{i j}=0
\end{gathered}
$$

and

$$
v_{12}^{V I}(x, t)=-\int_{-\infty}^{t} C_{1122}\left(x, t-t^{\prime}\right) E\left(x, t^{\prime}\right) \mathrm{d} t^{\prime}=-\int_{-\infty}^{t} E\left(x, t-t^{\prime}\right) C_{1122}\left(x, t^{\prime}\right) \mathrm{d} t^{\prime} .
$$

Note that for the Class VI PRs the loading path is defined by prescribing it as $\sigma_{11}(x)$. However, it must be remembered the this Class is based on the absolute neglect of the loading cycle and that a time independent stress is not realistically achievable for all times $t$.

Some of the ambiguities arising from the Class III PRs can be resolved, i.e. removed, by defining a Class VII PR as

$$
\overline{\bar{V}}_{1 j}^{V I I}(x, \omega) \underline{\underline{\operatorname{def}}}-\frac{\overline{\bar{C}}_{11 \underline{j j}}(x, \omega)}{\overline{\bar{C}}(x, \omega)} \Rightarrow C_{11 \underline{j j}}(x, t)=-\int_{-\infty}^{\infty} v_{1 j}\left(x, t-t^{\prime}\right) C\left(x, t^{\prime}\right) \mathrm{d} t^{\prime} \quad(j \neq 1)
$$

It is the only PR class that is entirely free from strain, stress and path restrictions since its definition is not based on the legacy Class I [2] [3], formulation or on any of its derivative definitions of Classes II to VI.

Comment 5. The Class VII is an artificially defined isothermal viscoelastic $P R$ devoid of strain or stress associations and hence path independent while still remaining a time dependent universal martial property. It obeys the construction mandated by Eqs. (32) necessary for the existence of EVCPs. This Class PR should, therefore, be the one used if it is desired to express constitutive relations in terms of PRs. However, since this $P R$ form necessitates a priori knowledge of two compliances that completely characterize the isotropic viscoelastic medium, it is fair to ask why bother with the PR. Furthermore, since Class VII PRs do not involve strains they are not experimentally measurable.

At least three more than rhetorical questions remain:

1) What, if any, is the physical meaning of the Class VI PRs, $v_{12}^{V I}$ ?

2) What is their relation to the strains?

3) What is the physical meaning, if any, of the double convolution integrals of Eqs. (32a) and (32c)?

The answers to all three questions are "none" indicating that they cannot be 
obtained directly through experimentally determined strains and stresses. Furthermore, due to the presence of a triple product in the above constitutive relations these PRs are not a portal to EVCP formulations. Even though the Class VI PRs appear as pure universal material property descriptors their utility function is less than that of its parts, i.e. the compliances, since these PRs are first and foremost process dependent and, therefore, restricted and limited in scope in this case to time independent 1-D stresses $v_{12}^{V I}$. (See the next Section on starting loading sequences to achieve time independent stresses, strains, their time rates, etc., in a dissipative material with memory effects.)

There is a certain irony accompanying the creation of the Class VI PR based on a time independent 1-D stress field

$$
\begin{aligned}
& \sigma_{11}(x, t)=\sigma_{11}(x) \text { all other } \sigma_{i j}(x, t)=0 \quad 0 \leq t \leq \infty \\
& \text { and } \sigma_{11}(x, t)=0 \quad-\infty \leq t \leq 0
\end{aligned}
$$

The intent is to produce a viscoelastic look alike to the structure of the elastic constitutive relations containing a mixture of moduli, stresses and the original Class I elastic PR [3]. What emerges as a Class VI PR is a function independent of strains and their history and one that is only dependent on certain moduli and compliances. Of course, in the elastic case the same path following Eqs. (31) leads to for $\sigma_{11}(x) \neq 0$ and all other $\sigma_{i j}=0$

Class VI elastic $\sigma_{11}^{E}(x, t)$ all other $\sigma_{i j}^{E}=0 \Rightarrow$

$$
v_{12}^{E V I}(x)=-\frac{C_{1122}^{E}(x)}{C^{E}(x)}=-\frac{C_{1122}^{E}(x) \sigma_{11}^{E}(x, t)}{C^{E}(x) \sigma_{11}^{E}(x, t)}=-\frac{\epsilon_{22}^{E}(x, t)}{\epsilon_{11}^{E}(x, t)}=v_{1-\mathrm{D} \text { loading only }}^{\substack{\text { original PR } \\ \text { Class } \mathrm{I}}}
$$

For a viscoelastic medium, $v_{12}^{V I}$ can be similarly interpreted as

Class VI viscoelastic $\sigma_{11}(x)$ all other $\sigma_{i j}=0 \Rightarrow$

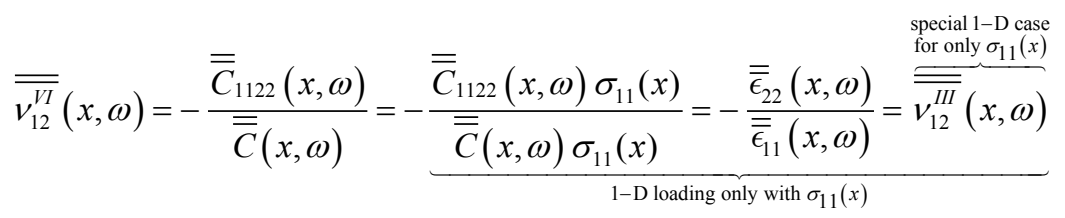

with the real time convolution integral property

$$
\epsilon_{22}(x, t)=-\int_{-\infty}^{t} v_{12}^{V I}\left(x, t-t^{\prime}\right) \epsilon_{11}\left(x, t^{\prime}\right) \mathrm{d} t^{\prime} \quad \text { with } \sigma_{11}(x) \neq 0 \text { and } \sigma_{\underline{j i}}=0 \text { for } j \neq 1 .
$$

It should be noted that the Class VI PR is a special case of the general Class III PR with $\sigma_{11}(x) \neq 0$ and $\sigma_{22}=\sigma_{33}=0$. Neither Class III nor Class VI PRs have a physical definition whose values could be measured experimentally in the

${ }^{9}$ For non-auxetic elastic materials, $C_{1122}^{0}<0$ and $C_{1122}(x, t)<0$ while $E(x, t)>G(x, t)>0$. For auxetic materials the reverse inequalities hold and the elastic PR's are in the range $-1 \leq v_{12}^{E}<0$. However, viscoelastic PRs have no such restrictions except at $t=0$, since the ICs are elastic conditions. 
time domain. While it is certainly possible to measure the strains $\epsilon_{i j}(x, t)$ in real time, the time functions associated with this PR class are complicated and the evaluation of the integral in (41) is difficult. The second integral of (32) clearly represents the proper form of the triple FT product involving PRs in the time domain. The character of the time function defining $v_{12}^{V I}(x, t)$ can be deduced from the inversion of the FTs, Eq. (36). Since the Prony series for $C_{1122}$ and $C$ are known expressions determined from generalized Kelvin or Maxwell models. However, since Eq. (36) requires the a priori determination of the compliances, one can justifiable inquire why bother with $v_{12}^{V I}$ when the characterization is complete with the knowledge of either set of the $C_{i j k l}$ or $E_{i j k l}$. Particularly, since detailed knowledge of these non-universal PRs still does not produce a useful EVCP and either the moduli or the compliances do so handily.

In the viscoelastic constitutive relations there appears to be no clear protocol to derive directly in real time space either of the first two versions of double convolution integrals displayed in (32) that can be based on thermodynamic first principles or any mechanical or electrical simulation model generated under the three fundamental thermodynamic laws or on the Boltzmann superposition principle [6]. Only through mimicking Hooke's law in the FT space can Eqs. (32) be so postulated in an inverse fashion. In a larger sense then, the intro- duction of PRs into viscoelastic stress-strain relations can be interpreted as an artificial act of faith not based on first principles, i.e. with PRs related to thermodynamic derivatives, or even to achievable physical characterizations and experiments, as will be seen subsequently.

Comment 6. None of the five PR categories defined in [87] [88] [89] fit these needed real time prescriptions, nor does the sixth class fit the constraints imposed by (32). Consequently, it would appear that no universal EVCP based on PRs can be rationally deduced or justified.

The expressions (26) are valid in a very limited sense for Class II PRs $\overline{\bar{v}}^{I I}(x, \omega)$, but are only applicable there and cannot be exported to the general Class I expressions (24) with $\overline{\bar{v}}(x, \omega)$ and, therefore, are of no use beyond the one and only case of a time independent strain component, say $\epsilon_{11}(x)$, (see next Section). Additionally, any expressions for $\overline{\bar{G}}$ and $\overline{\bar{K}}$ derived from (30) based on Class II PRs $v^{I I}$ apply only to conditions of time independent strains $\epsilon_{11}^{0}$ or $\epsilon_{11}(x)$ and cannot be generalized to any other strain field. Their complete lack of universality makes such modulus expressions useless from a practical point of view. Similar universal compliance (and modulus) relations may be found in Refs [97]-[102].

\subsection{Comments on PR's Dependence on Time}

The following is a partial list of the cardinal difficulties that are directly attributable to their time dependence and that are associated with the use of viscoelastic PRs:

1) At least seven mutually independent PR definitions are available. 
2) Difficulties stemming from the improper assembly of constitutive relations containing PRs in the time space resulting in combinations

$$
\begin{aligned}
I N T_{1}(t) & =\int_{-\infty}^{t} E_{1122}^{*}\left(t-t^{\prime}\right) \epsilon_{22}\left(t^{\prime}\right) \mathrm{d} t^{\prime}=-\int_{-\infty}^{t}\left\{\int_{-\infty}^{t^{\prime}} v^{I I I}\left(t^{\prime}-s\right) E^{*}(s) \mathrm{d} s\right\} \epsilon_{11}\left(t-t^{\prime}\right) \mathrm{d} t^{\prime} \\
& =-\int_{-\infty}^{t}\left\{\int_{-\infty}^{t^{\prime}} v^{I I I}(s) E^{*}\left(t^{\prime}-s\right) \mathrm{d} s\right\} \epsilon_{11}\left(t-t^{\prime}\right) \mathrm{d} t^{\prime}
\end{aligned}
$$

for which no integral transform can obtained that leads to a proper EVCP expression. See Eqs. (33) for proper time space forms required to realize the needed double integral transform products and triple time function products (32).

3) Conversely independent assemblies in the integral transform space (FT and LT) lead to some combinations displayed in Eqs. (32) such that the triple products have no physical counterpart in the real time space.

4) Additionally and separately viscoelastic PRs are also stress and stress history (i.e. path) dependent.

\subsection{The Physics of Time Invariant Stresses, Strains and of Their Derivatives}

The Influence of Constant Stress/Strain Models on PRs

The Class II and VI PR formulations are predicated on the mathematically correct prescription of constant 1-D strain (II) or constant 1-D stress (VI), such that

$$
\begin{array}{lll}
\text { Class } I I \Rightarrow \epsilon_{11}(x, t)=H(t) \epsilon_{11}^{o} & 0 \leq t \leq \infty \\
\text { Class } V I \Rightarrow \sigma_{11}(x, t)=H(t) \sigma_{11}^{o} & 0 \leq t \leq \infty
\end{array}
$$

where $H(t)$ is the Heaviside step function with defined properties [106]

$$
H(t)= \begin{cases}0 & t \leq 0 \\ 1 & t \geq 0\end{cases}
$$

The special $H(t)$ function has a discontinuity at $t=0$, but possesses a derivative i.e. another special function-the Dirac delta function or impulse function [126]

$$
\frac{\mathrm{d} H(t)}{\mathrm{d} t}=\delta(t)=\left\{\begin{array}{ll}
\lim _{a \rightarrow 0}\left\{\frac{1}{2 a}\right\} \rightarrow \infty & t=0 \\
0 & t \neq 0
\end{array} \text { with } \int_{-\infty}^{\infty} \delta(t) \mathrm{d} t=1\right.
$$

Consequently the Class II and VI mathematical models are well defined but loading (path) dependent. Unfortunately the actual physics of this loading problem are considerably less forgiving, as shown analytically in [93] [94] and experimentally in [97]-[104]. An instantaneous displacement requires an infinite acceleration and an associated infinite force in zero time with similar physically unattainable conditions for constant stress and strain rates. Further as analytically proved in [127], even the analytical modeled application of Dirac delta function loads lead to singularities in time derivatives of displacements for linear and nonlinear elastic and viscoelastic media.

In addition, testing machine crossheads have inertia and require finite times 
to develop displacements and loads as demonstrated in [103]. In Section 4 data is displayed for experimental loading cycle results of high polymer tension coupons obtained from four different INSTRON machines. The detailed analyses show that the rise times to constant strain rates actually occur considerably after creep and relaxation have begun at 0.001 to $0.01 \mathrm{sec}$, consequently rendering the mathematical model of Class II and VI physically unrealizable and, therefore, making the associated PR functions physically unachievable and, therefore, unacceptable. Furthermore, in [95] [96] analytical studies clearly show the heavy influence of various loading protocols on the accuracies of viscoelastic material characterization.

\subsection{Penalties Associated with Instantaneous Loading Models}

\subsubsection{Dynamic Loadings}

The instantaneous loading concept can be modeled mathematically [27] through the use of Dirac delta $\delta(t)$ and Heaviside $H(t)$ functions [106] and [127] while the gradual loading process to achieve a steady or time dependent loads requires more than one functional representation For instance, consider the case of dynamic loadings leading to wave propagations. Figure 4 illustrates the experimentally measured 1-D effects of an impact load on a bar at $(0, t)$. In particular note the green curve which is the impact force. The other two curves represent accelerometer readings at two distinct points at $x_{1}>0$. The impact loading, then, can be represented schematically as in Figure 5 and by Eqs. (46) to (48)

$\left.\begin{array}{l}\text { instantaneous loading, } \\ \text { mathematical model }\end{array}\right\} \Rightarrow \sigma_{11}(0, t)=\sigma_{11}^{0} \delta(t) \quad 0 \leq t \leq \infty$

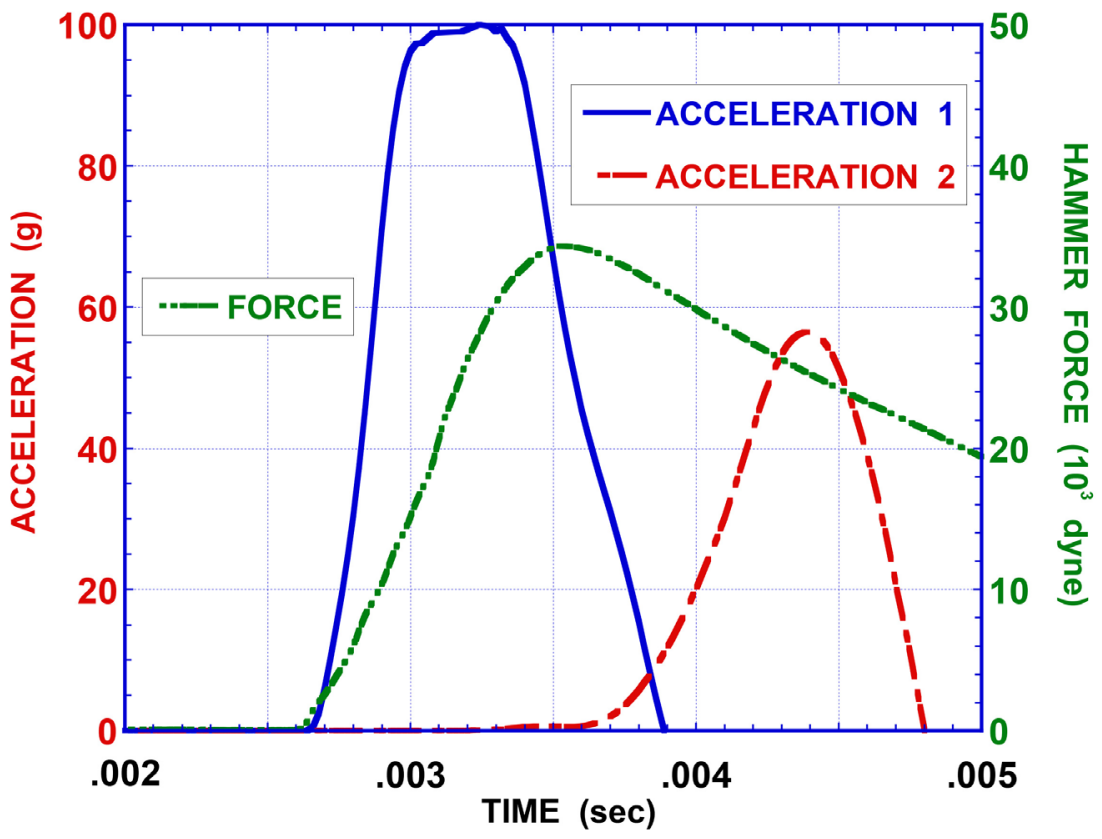

Figure 4. Impulse force and accelerometer responses [104]. 


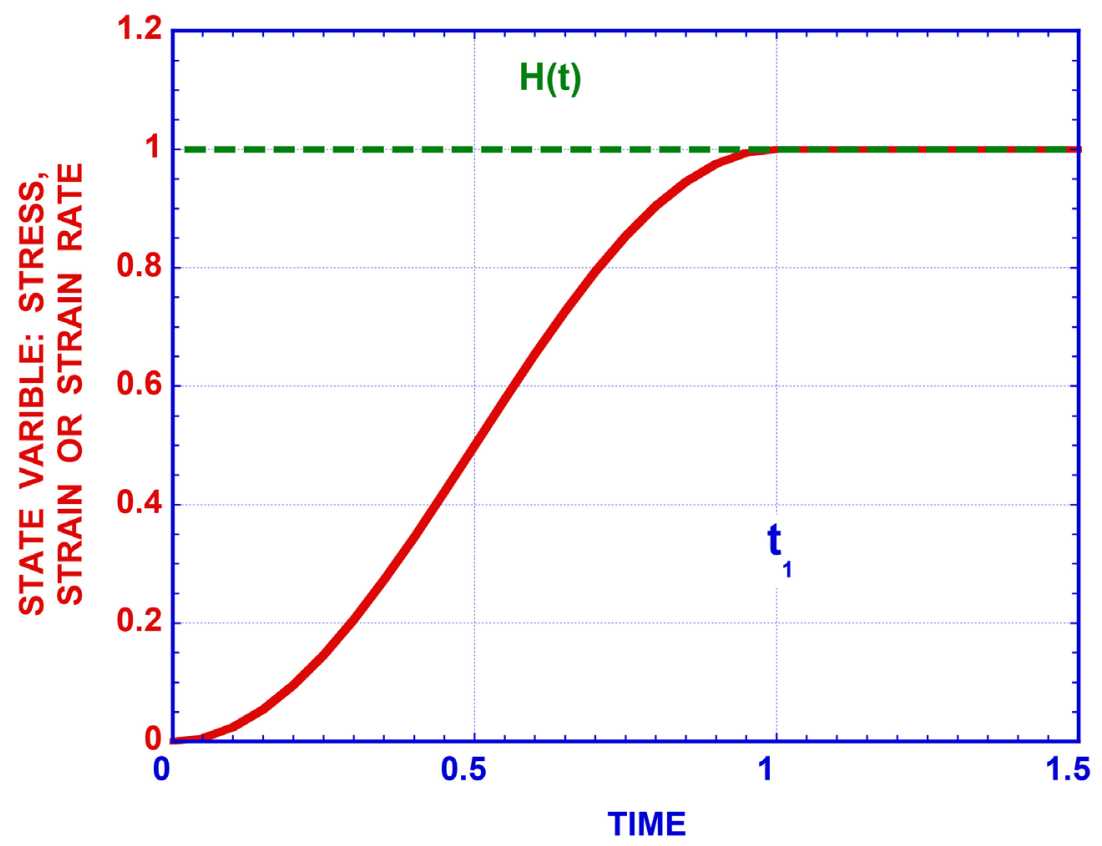

Figure 5. Initial instantaneous and gradual loading phase schematic.

$$
\left.\begin{array}{l}
\text { gradual loading, } \\
\text { physical model }
\end{array}\right\} \Rightarrow \sigma_{11}(0, t)= \begin{cases}0 & t \leq 0 \\
\sigma_{11}^{0} f_{\sigma}(t) & 0 \leq t \leq t_{2} \\
0 & t_{2} \leq t \leq \infty\end{cases}
$$

with four conditions on $f(t)$

$$
f_{\sigma}(0)=\left.\frac{f_{\sigma}(t)}{\mathrm{d} t}\right|_{t=0}=f_{\sigma}\left(t_{2}\right)=\left.\frac{f_{\sigma}(t)}{\mathrm{d} t}\right|_{t=t_{2}}=0
$$

The inherent difficulties associated with the $\delta(t)$ and $H(t)$ functions are due to the latter's double values and hence discontinuities at $t=0$. Consider their troublesome properties

$$
\begin{aligned}
& \lim _{t \rightarrow 0} \delta(t) \rightarrow \infty, \quad \frac{\mathrm{d}^{n} \delta(t)}{\mathrm{d} t^{n}}=(-1)^{n} n ! \frac{\delta(t)}{t^{n}}, \\
& \frac{\mathrm{d} H(t)}{\mathrm{d} t}=\delta(t), \quad \lim _{t \rightarrow 0} \int_{-\infty}^{\infty} \frac{1}{t} \frac{\mathrm{d} \delta(t)}{\mathrm{d} t} d t \rightarrow \infty
\end{aligned}
$$

plus those of (45) and the more beneficial finite one

$$
\lim _{t \rightarrow 0} \int_{\infty}^{\infty} \delta(t) \mathrm{d} t \rightarrow 1
$$

This effectively means that for the mathematical model, if the IC properly consists of an instantaneous impulse force

$$
\sigma_{11}(0,0)=\left.\sigma_{11}^{0} \delta(t)\right|_{t=0} \rightarrow-\infty
$$

then it will propagate into the linear and nonlinear elastic and viscoelastic displacement solutions, such that $u_{1}(x, t) \propto H(t)$ making the temporal derivatives that determine velocities $\dot{u}_{1}(x, t)$ and accelerations $\ddot{u}_{1}(x, t)$ unbounded 
at $t=x_{1 / c}$ when physically both are finite [127].

In view of consistency requirements one should expect no less than an infinite acceleration to produce an instantaneous impulse force $\sigma_{11}(0,0)$.

\subsubsection{Quasi-Static Loadings: Creep, Relaxation, “Constant” Strain Rate, SHM}

By contrast the quasi-static models differ from that dynamic ones essentially in the fact that after the completion of the time dependent loading cycle they remain at a constant value. Let $\boldsymbol{S} \boldsymbol{V}(x, t)$ represent the set of state variables, such that $\boldsymbol{S} \boldsymbol{V}=\left\{\sigma_{i j}, \epsilon_{i j}, \dot{\epsilon}_{i j}, u_{i}, \cdots\right\}$. Then the model representation become

$$
\begin{aligned}
& \left.\begin{array}{l}
\text { instantaneous loading, } \\
\text { mathematical model }
\end{array}\right\} \Rightarrow \boldsymbol{S} \boldsymbol{V}(0, t)=\boldsymbol{S} \boldsymbol{V}^{0} H(t) \quad 0 \leq t \leq \infty \\
& \left.\begin{array}{l}
\text { gradual loading, } \\
\text { physical model }
\end{array}\right\} \Rightarrow \boldsymbol{S} \boldsymbol{V}(0, t)= \begin{cases}0 & t \leq 0 \\
\boldsymbol{S} \boldsymbol{V}^{0} f_{S V}(t) & 0 \leq t \leq t_{1} \\
\boldsymbol{S} \boldsymbol{V}^{0} H\left(t-t_{1}\right) & t_{1} \leq t \leq \infty\end{cases}
\end{aligned}
$$

With the mathematical model, one is again faced with a discontinuity due to the double valued Heaviside function at the temporal origin resulting in

$$
\lim _{t \rightarrow 0}\left\{\frac{\partial[\boldsymbol{S} \boldsymbol{V}(0, t)]}{\mathrm{d} t}\right\}=\lim _{t \rightarrow 0} \delta(t) \rightarrow \infty
$$

Similar conclusions to those enunciated in Section 3.7.1 apply here as well.

Comment 7. In the final analysis the impulsive loading and sudden displacement initial problems are reminiscent of the non-existing sharp edged gusts, instantaneous penetration of atmospheric disturbances and zero time lift and drag buildups encountered in aeroelasticity. All such aerodynamically improbable physical models have been effectively corrected by the introduction of the three distinct delay functions of Theodorsen, Küssner and Wagner [128]. In equal measures, the solid mechanics physical aspects command that no less be done for the preceding ICs and possible subsequent changes in loading functions.

\subsection{Analysis of Time Dependent Experimental Ramp Loadings}

Refs. [92] and [93] contain analytical simulations that show the various regions of viscoelastic moduli, and conversely of compliances. The simulation studies proved that the ratio $E_{0} / E_{\infty}$ and the extent as well as the slope of Region $\mathcal{C}$ are the most important contributors to optimum viscoelastic material damping properties as seen in Figure 6. Of course, the shape and regions of the relaxation modulus curves are directly dependent on the sensitivity of the experiments that are used to determine such modulus properties. Furthermore, since PRs are related to moduli as seen in Eqs. (28) and (32) they exhibit similar sensitivities.

In Ref. [95] a systematic analysis of starting transients influence on constitutive relations is undertaken by analytically investigating simulation models for simple tension experiments. Since numerical values of material parameters are 


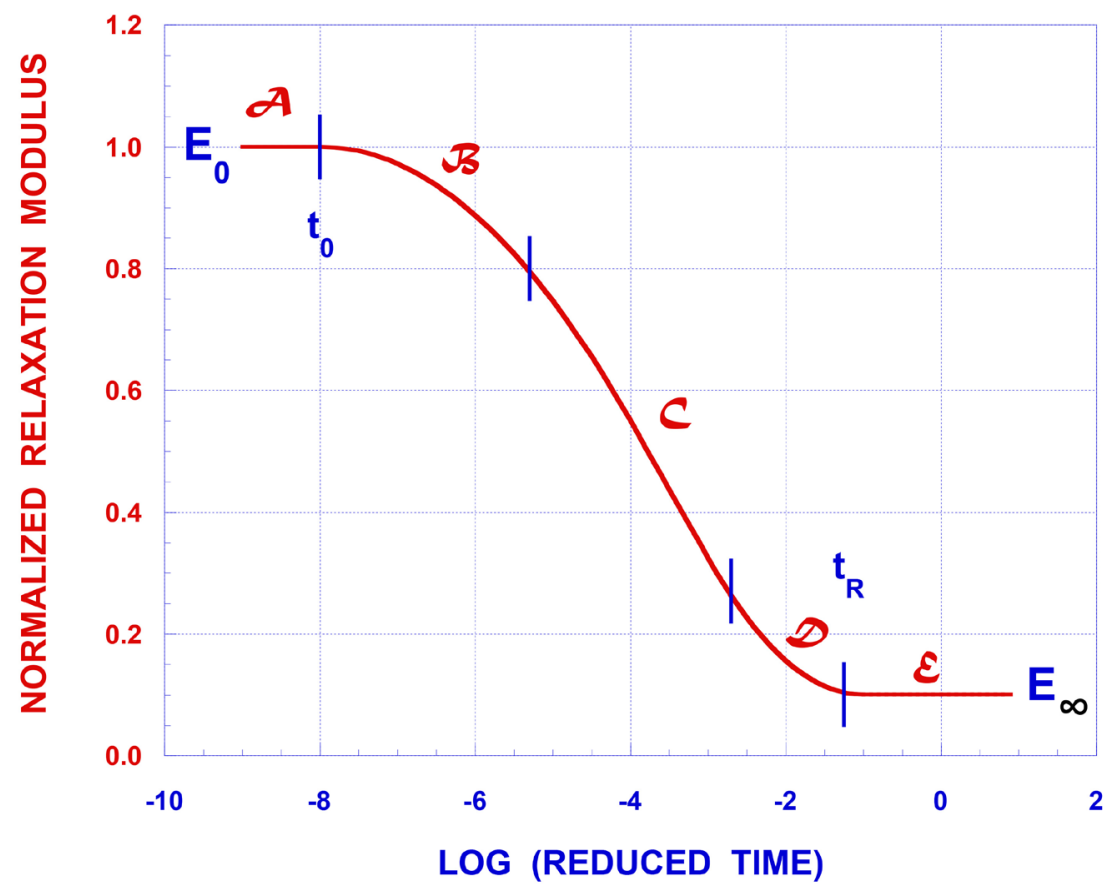

Figure 6. Viscoelastic modulus regions [92].

sought, meaningful experiments need to be devised which can be solved analytically with symbolic values for the as yet unknown material parameters. Consider a "simple" 1-D tension or compression (without buckling) creep experiment (Figure 4). In a Cartesian coordinate system $x=x_{i}$ with $i=1,2,3$ and $x_{1}$ the loaded direction. Whether or not shear is present at $x_{1}=0$ in the $x_{2}$ direction has no bearing on the loading function formulation, but, of courses, influences internal stress distributions.

The loading function $F(t)$ is defined in three time domains as

$$
F(t)=\left\{\begin{array}{lll}
0 & t \leq 0 & \text { Initial Conditions (ICs) } \\
F_{0} f(t) & 0 \leq t \leq t_{1} & \text { loading path with ramp function } f(t) \\
F_{0} H\left(t-t_{1}\right) & t \geq t_{1} & \text { steady state loading }
\end{array}\right.
$$

where $F_{0}$ is a constant amplitude, i.e. $\dot{\epsilon}_{11}^{0}$ or $\dot{\sigma}_{11}^{0}$ of (43), and $f(t)$ is one of the following functions

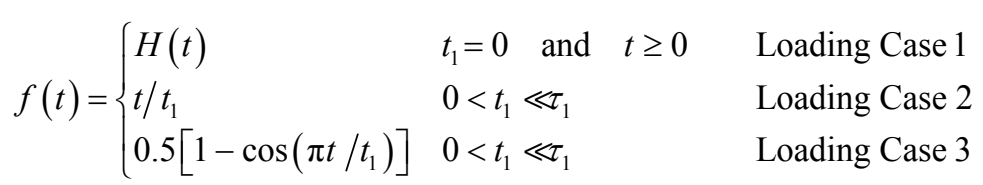

with $H(t)$ the Heaviside unit step function. While mathematically all three functions are admissible, the only physically "reasonable" function is the third one, although other similar forms are equally acceptable, as it properly defines a gradual load rise from 0 to unity with physically required vanishing slopes at $t=0$ and $t=t_{1}$.

The time $t_{1}$ necessary to achieve the constant load $F_{0}$ is dictated by laboratory equipment used to induce loadings. While $t_{1}$ has no predetermined re- 
lation to $t_{0}$, the time when relaxation begins, i.e. the relaxation modulus $E\left(t_{0}\right)=E^{E} \quad$ (the instantaneous elastic Young's modulus) for $t<t_{o}$ and $E(t)<E^{E}$ for $t>t_{0}$, the determination of moduli values are strongly influenced by the relative position of $t_{0}$ and $t_{1}$.

The next two figure from [95] present creep functions determined by solving the governing relations including the loading phase for a given load case and different values of the loading time $t_{1}$. For illustration purposes the strain curves were established prescribing that the material characteristics are known. The results obtained following the proposed procedure were compared to this reference curve. Times $t_{1}$ were chosen here by evaluating the increase in $\psi\left(t_{1}\right)$ compared to $\psi_{0}$ and are indicated as percentages in the plot legends. Since the values $t_{1}$ are not significant on their own, they will be identified on the plots by the relative increase in $\psi\left(t_{1}\right)$ from $\psi_{0}$.

Figure 7 and Figure 8 from Ref. [95] represent creep curves obtained through analytical simulations by assuming that the loading conforms to an ideal situation, i.e. by replacing $f(t)=H(t)$ by other loading functions as detailed in (55) and (56). If the strain values used when solving the system of equations correspond indeed to Loading Case 1, the creep curve is recovered with good accuracy (less than $0.5 \%$ error for $N=33$ terms in Prony series). However, if a more realistic situation is considered (Loading Cases 2 and 3), the material characteristics are determined with some error depending on $t_{1}$. In Figure 7 the strains were obtained from Loading Case 2 and all the points $t<t_{1}$ were left out of the computation. As expected, the largest deviation appears in the values of $\psi_{0}=C^{0}$. Almost identical results were obtained using strains corresponding to Loading Case 3 and displayed in Figure 8. A number of additional examples may be found in [95]. The influence of testing machine cross head speeds is discussed in the next Section.

\subsection{The Influence of the Loading Phase on Constitutive Relations and PRs}

Starting with the linear anisotropic viscoelastic constitutive relations (16) and specializing them to 1-D loading isothermal isotropic conditions yields

$$
\begin{aligned}
\epsilon_{11}(x, t)-\underbrace{C_{0} \sigma_{11}(x, t)}_{\begin{array}{c}
\text { instantaneous } \\
\text { elastic response }
\end{array}} & =\underbrace{\int_{0}^{t} C^{*}\left(t-t^{\prime}\right) \sigma_{11}\left(x, t^{\prime}\right) \mathrm{d} t^{\prime}}_{\text {viscoelastic response }} \\
& =\underbrace{\int_{0}^{t} \sum_{n=1}^{N} \frac{C_{n}}{\tau_{n}} \exp \left(-\frac{t-t^{\prime}}{\tau_{n}}\right) \sigma_{11}\left(x, t^{\prime}\right) \mathrm{d} t^{\prime}}_{\text {Prony series representation }}
\end{aligned}
$$

For a hypothetical constant stress $\sigma_{11}^{0}$ as defined by (43), Eq. (57) can be integrated to yield

$$
\begin{aligned}
& \frac{\epsilon_{11}^{\text {approx }}(t)}{\sigma_{11}^{0}}=C_{0}+\sum_{n=1}^{N} C_{n}\left[1-\exp \left(-\frac{t}{\tau_{n}}\right)\right]=C(t) \\
& \text { with } \sigma_{11}(t)=\sigma_{11}^{0} \text { for } 0 \leq t \leq \infty
\end{aligned}
$$

where 


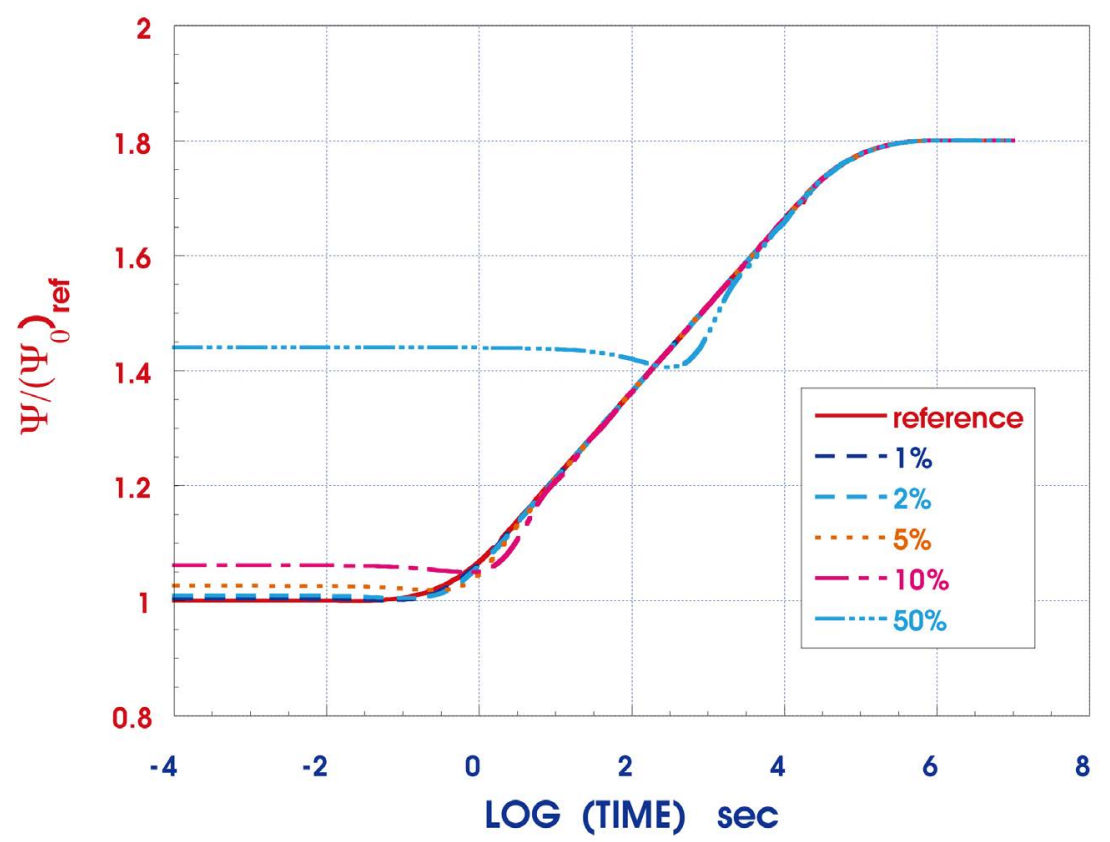

Figure 7. Loading Case 2 creep function determined assuming loading Case 1 [94].

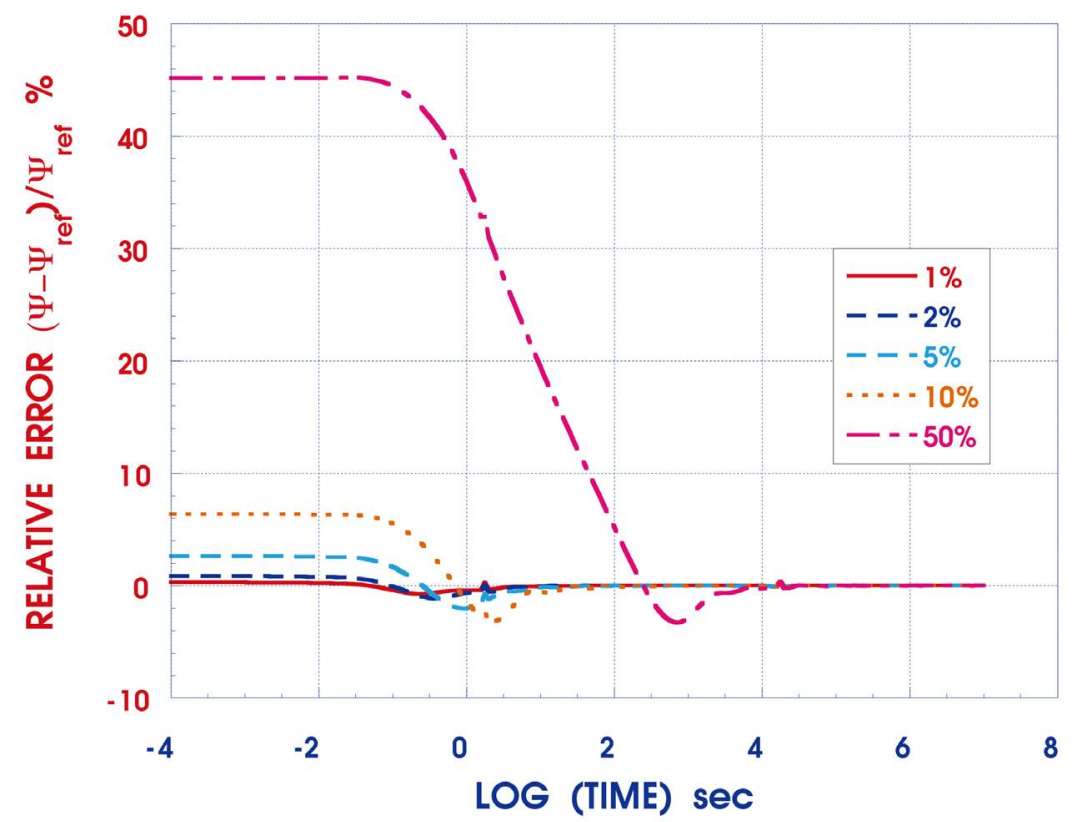

Figure 8. Loading Case 3 Creep Function determined assuming loading Case 1 [95].

$$
C_{\infty}=C_{0}+\sum_{n=1}^{N} C_{n}
$$

Eq. (58) is correct if and only if $\sigma_{11}(t)=0$ for $t \leq 0$ and $\sigma_{11}(t)=\sigma_{11}^{0}$ and all other $\sigma_{i j}(t)=0$ for all $t \geq 0$. Therefore, it does not apply when the loading phase (55) is taken into account since then Eq. (57) is no longer correct and must be properly interpreted.

If the loading cycle (55) is taken into account then Eq. (57) transforms into 


$$
\epsilon_{11}(t)-\underbrace{C_{0} \sigma_{11}(t)}_{\begin{array}{c}
\text { instantaneous } \\
\text { elastic } \\
\text { response }
\end{array}}=\underbrace{\int_{0}^{t_{1}} C^{*}\left(t-t^{\prime}\right) \sigma_{11}\left(t^{\prime}\right) \mathrm{d} t^{\prime}}_{\text {loading phase }}+\underbrace{\int_{t_{1}}^{t} C^{*}\left(t-t^{\prime}\right) \sigma_{11}\left(t^{\prime}\right) \mathrm{d} t^{\prime}}_{\text {quasi-static or dynamic phase }}
$$

and

$$
\frac{\epsilon_{11}(t)}{\sigma_{11}^{0}} \neq C(t) \text { with } \quad C^{*}\left(t-t^{\prime}\right)=\frac{\partial C\left(t-t^{\prime}\right)}{\partial t^{\prime}}
$$

Therefore, the error in strain responses generated by disregarding the starting stress transients is given by

$$
\operatorname{err}_{\epsilon_{11}}(t)=1-\frac{\epsilon_{11}^{\text {approx }}(t)}{\epsilon_{11}(t)}
$$

and is shown in Figure 9 for experimental data obtained in [105]. These results show that the effects of the starting transients decay with time but that they also represent important contributions to inaccuracies over significant time periods in excess of $t_{1}$, the time to reach steady-state loading conditions.

However, a much more compelling case can be made for the linear relaxation/creep functions or moduli/compliances, which are more pervasive and universal properties than specific strain responses [95] [96].

\subsection{The Case III PR Caveat}

The Class III PR integral transform definition, Table 3, while lending itself more expeditiously to the formation of an EVCP has troublesome idiosyncrasies and a dichotomy of meanings in the time space. Note that for Case III PRs, for 1-D loadings and time independent temperatures the convolution integrals prevail and then and only then

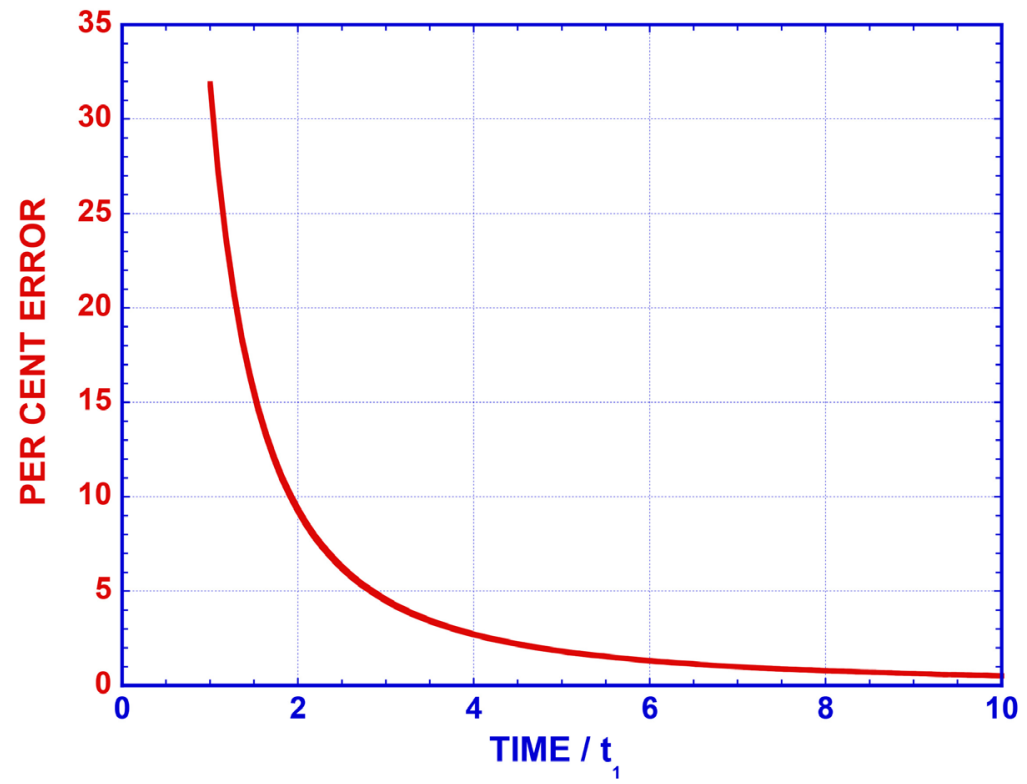

Figure 9. Error in viscoelastic strains by disregarding starting load transients for data from [105]. 


$$
\overline{\bar{v}}_{12}^{I I I}(x, \omega)=-\frac{\overline{\bar{\epsilon}}_{22}(x, t)}{\overline{\bar{\epsilon}}_{11}(x, t)}=-\frac{\overline{\bar{C}}_{1122}(\omega) \overline{\bar{\sigma}}_{11}(\omega)}{\overline{\bar{C}}_{1111}(\omega) \overline{\bar{\sigma}}_{11}(\omega)}=-\frac{\overline{\bar{C}}_{1122}(x, \omega)}{\overline{\bar{C}}(x, \omega)} \quad 1-\text { D loading only }
$$

which makes the expression independent of $\overline{\bar{\sigma}}_{11}(x, \omega)$ but not of the 1-D loading process (path) that specifically creates it. Eq. (63) does not apply to multiple loadings where it becomes a function of all the pertinent normal stresses $\overline{\bar{\sigma}}_{i i}$, i.e.

$$
\overline{\bar{V}}_{12}^{I I I}(x, \omega)=-\frac{\overline{\bar{\epsilon}}_{22}(x, \omega)}{\overline{\bar{\epsilon}}_{11}(x, \omega)}=-\frac{\sum_{i=1}^{3} \overline{\bar{C}}_{i i 22}(x, \omega) \overline{\bar{\sigma}}_{i i}(x, \omega)}{\sum_{i=1}^{3} \overline{\bar{C}}_{i i 11}(x, \omega) \overline{\bar{\sigma}}_{i i}(x, \omega)}
$$

with

$$
C(t)=C_{1111}(t)=C_{2222}(t)=C_{3333}(t) \neq C_{1122}(t) \text { and } C_{1122}(t)=C_{1133}(t)
$$

for isotropic materials.

The normal stresses $\sigma_{i i}(x, t)$ can be described by continuous time functions, provided the loading functions are not discontinuous. Then for multi-D loadings and general anisotropic linear viscoelastic media, the Class III PR is given by

$$
\overline{\bar{v}}_{i j}^{I I I}(x, t)=-\frac{\overline{\bar{\epsilon}}_{i j}(x, \omega)}{\overline{\bar{\epsilon}}_{i \underline{i}}(x, \omega)}=-\frac{\overline{\bar{C}}_{i j k l}(x, \omega) \overline{\bar{\sigma}}_{k l}(x, \omega)}{\overline{\bar{C}}_{\underline{i} m n}(x, \omega) \overline{\bar{\sigma}}_{m n}(x, \omega)} \quad i \neq j
$$

Consequently, in all these instances the Class III PRs are also dependent on all non-zero stresses and on their histories as well as explicitly on time. Thus, different experimental determinations of PRs involving diverse stress and strain histories, lead to distinct PR functions for the same viscoelastic medium.

The neglect of the loading cycle in the period $0 \leq t \leq t_{1}$ leads to serious inaccuracies and discrepancies in response patterns particularly for slower loading rates as demonstrated analytically in [95] [96] and confirmed experimentally in [97]-[104]. See Eqs. (43)-(56) and Figure 2, Figure 7, Figure 8 and Figure 10, which are pertinent to the above discussion.

Matching analyses for constant $\epsilon_{11}^{0}, \dot{\epsilon}_{11}^{0}$ and $\dot{\sigma}_{11}^{0}$ for $t \geq 0$ can also be performed and similar conclusions can be reached for each of these loading cases.

Additionally, Category III PRs and their similar cousins carry with them an implicit contradiction. Consider a 1-D loading $\sigma_{11}(t)$, where this function is integrable for $t \in[-\infty, \infty]$. See Table 1 . Upon FT inversion one is lead to the limited relation

$$
C_{1122}(t)=-\int_{-\infty}^{t} v^{I I I}\left(t-t^{\prime}\right) C_{1111}\left(t^{\prime}\right) \mathrm{d} t^{\prime} \quad-\infty \leq t \leq \infty \quad 1-\mathrm{D} \text { loading only }
$$

when in fact it should read for $-\infty \leq t \leq \infty$, cf. Eq. (36).

$$
\underbrace{\int_{-\infty}^{t} C_{1122}^{*}\left(t-t^{\prime}\right) \sigma_{11}\left(t^{\prime}\right) \mathrm{d} t^{\prime}}_{=\epsilon_{22}(t)}=-\int_{-\infty}^{t} v^{I I I}\left(t-t^{\prime}\right) \underbrace{\left\{\int_{-\infty}^{t^{\prime}} C_{1111}^{*}\left(t^{\prime}-s\right) \sigma_{11}(s) \mathrm{d} s\right\}}_{=\epsilon_{11}(t)} \mathrm{d} t^{\prime}
$$




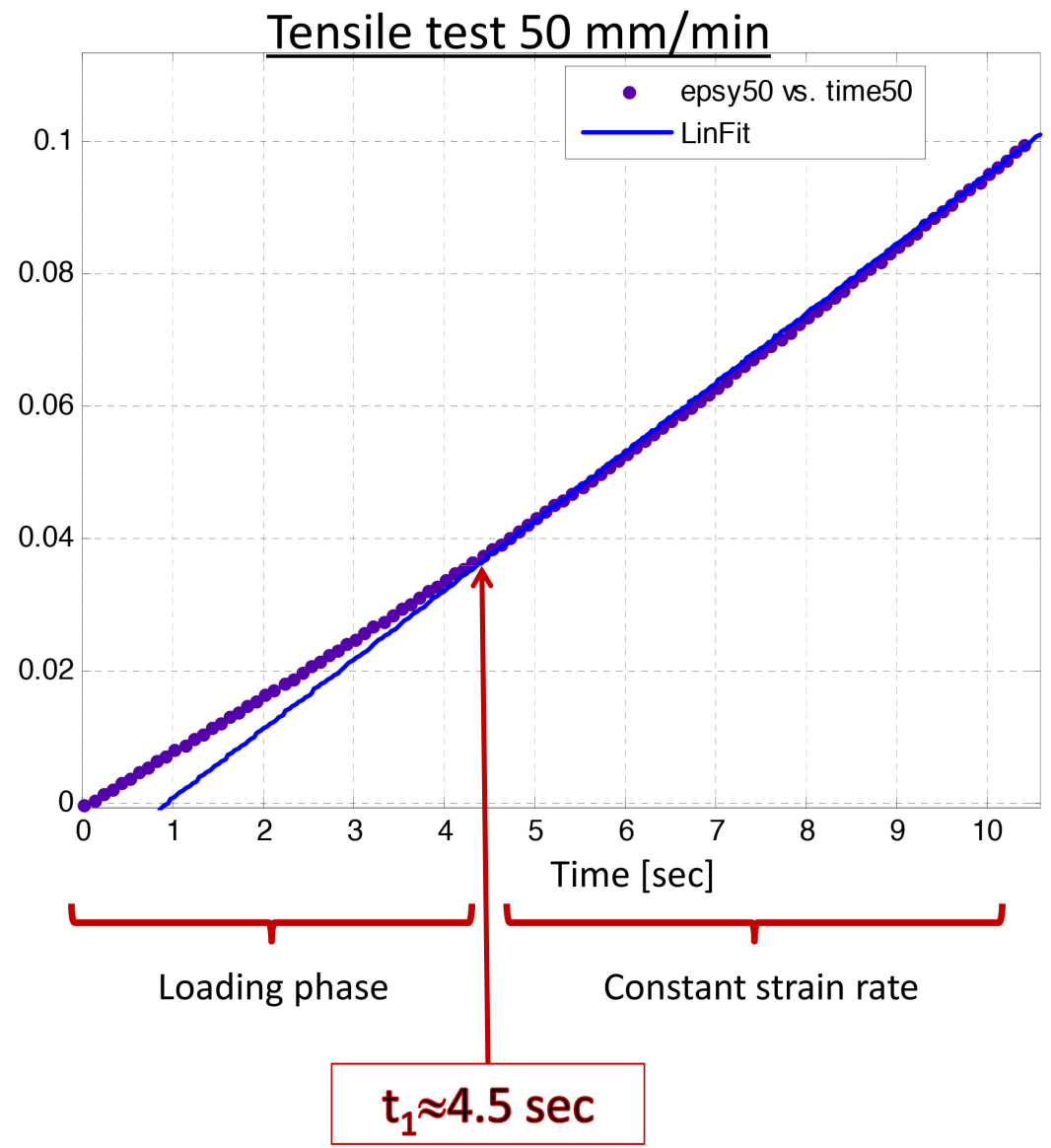

Figure 10. Time to reach constant strain rate of $50 \mathrm{~mm} / \mathrm{min}$ [103].

or

$$
\int_{-\infty}^{t} C_{1122}\left(t-t^{\prime}\right) \frac{\partial \sigma_{11}\left(t^{\prime}\right)}{\partial t^{\prime}} \mathrm{d} t^{\prime}=-\int_{-\infty}^{t} v^{I I I}\left(t-t^{\prime}\right)\left\{\int_{-\infty}^{t^{\prime}} C_{1111}\left(t^{\prime}-s\right) \frac{\partial \sigma_{11}(s)}{\partial s} \mathrm{~d} s\right\} \mathrm{d} t^{\prime}
$$

Eqs. (67) to (69) are identical if and only if the loading is 1-D and the stress is limited to $\sigma_{11}(x)$, i.e. a time independent stress component, which leads to significant characterization errors [95] [96]. In either exact or approximate representation Class III PRs are, therefore, also stress history (path, process) dependent.

Furthermore, if the constitutive relations are of the linear non-convolution type then for the 1-D loading

$$
\overline{\bar{V}}_{21}^{I I I}(x, \omega)=-\frac{\int_{-\infty}^{\infty} \int_{-\infty}^{t} C_{1122}\left(x, t, t^{\prime}\right) \frac{\partial \sigma_{11}\left(x, t^{\prime}\right)}{\partial t^{\prime}} \mathrm{d} t^{\prime} \exp (-l \omega t) \mathrm{d} t}{\int_{-\infty}^{\infty} \int_{-\infty}^{t} C\left(x, t, t^{\prime}\right) \frac{\partial \sigma_{11}\left(x, t^{\prime}\right)}{\partial t^{\prime}} \mathrm{d} t^{\prime} \exp (-l \omega t) \mathrm{d} t}
$$

and this PR is clearly implicitly stress and stress history (path) dependent, since generally $C(t)=C_{1111}(t) \neq C_{1122}(t)$. The non-convolution phenomenon takes place under the frequent time dependent temperature conditions when $T(x, t)$ and then 


$$
C_{i j k l}\left[x, t-t^{\prime}, T\left(x, t^{\prime}\right)\right]=C_{i j k l}\left(x, t, t^{\prime}\right)
$$

In summary, it is seen that

1) Eq. (63) is needed for the constructions of an EVCP in the transform space and its 1-D form is stress independent

2) Eq. (69) is the proper form of the constitutive relations in the real time space and is stress and stress history dependent

3) Proper care must be exercised in using and interpreting Eqs. (63) and (69) since they represent contradictory representations

Comment 8. While the preceding analyses establish Class III PR protocols that lead to the EVCP, they provide no clues as to what the proper accompanying $P R$ functions are. The $P R$ analysis is built on the superposition of responses to $1-D$ loading $(s)$ without specification as what it they should be, i.e. constant stress, strain, their time derivatives, etc. It has been established that viscoelastic PRs, unlike moduli and compliances, are time, stress and stress history (path) dependent and hence non-unique. This ambiguity renders viscoelastic PRs unsuited for general material characterization. Consequently, constitutive relations and EVCPS based on universal path independent moduli and/ or compliances remain the material descriptors of choice.

\section{Discussion}

In Figure 1, the Class I PRs for the same material are presented for four distinct experimental loading conditions ( 2 relaxation and 2 creep experiments) but with identical specimen, types of experimental loadings and boundary conditions [102]. Calculated from Eq. (1) and based on actual measured strains, the resulting PRs for these four different 1-D loadings exhibit time variations that are significantly distinct to testify to the viscoelastic PR inability to be classified as a unique material property due to its strong dependence on loading histories even in relatively small strain and short time ranges. In the two relaxation experiments and for over $95 \%$ of the operating time intervals, the viscoelastic PR values exceed 0.5, which is the positive upper limit for linear elasticity. In the creep experiment for the same material the PR values are all less than a half. The only difference is the loading rate and the PRs were computed directly from the measured strains, without regard to any a priori material property determinations. Similarly, the same experimental data from the four distinct loadings will yield four distinct curves of Class III PRs $\overline{\overline{v^{I I I}}}(\omega)$.

Similar differences in PR time function obtained in creep and relaxation were reported in [98].

Attempts at determining the time time $t_{1}$ when steady-state loadings are reached based on examination of strains as seen in Figure 9 have been disappointing and misleading [103]. This protocol produced physically indefensible results with erroneous trends [104]. On the other hand a new protocol based on strain accelerations $\partial^{2} \epsilon_{11}(t) / \partial t^{2}$ calculated from measured strains $\epsilon_{11}(t)$ 
produce physical results Figure 11 and Figure 12. However, the determination of moduli and/or compliances without PRs avoided any $t_{1}$ problems by numerically integrating the governing over the entire time range while specifically including the actual loading phases and measured strains [102] [103] [104].

Comment 9. The great universal utility of elastic Poisson's ratios due to their characteristically constant values for each homogeneous elastic material at a given temperature is lost in viscoelasticity. Conclusive experimental and analytical evidence undisputedly indicates that viscoelastic PRs are time, stress and stress history dependent and, therefore, not unique material descriptors. Consequently, they are not exportable or interchangeable from one loading condition to another.

Furthermore, the analysis demonstrates that the elastic-viscoelastic correspondence principle can only be expressed in terms of relaxation moduli or creep compliances and does not involve PRs. The equivalent triple product of Fourier transforms of PRs, moduli/compliances and strains/stresses necessary for the establishment of the EVCP, while mimicking Hooke's law, does not invert to a proper set of constitutive relations in the time space and, therefore, no EVCP involving PRs is possible.

Additionally and separately, analyses and experiments show that starting loading build ups, including achievable testing machine cross head speeds, are of significant importance to mandate their inclusion in material characterization protocols. Their neglect in the definitions of Class II and VI PRs, while mathematically defensible, are physically unattainable and unrealistic. Consequently, Class II and VI definitions only exist as idealized mathematical models but

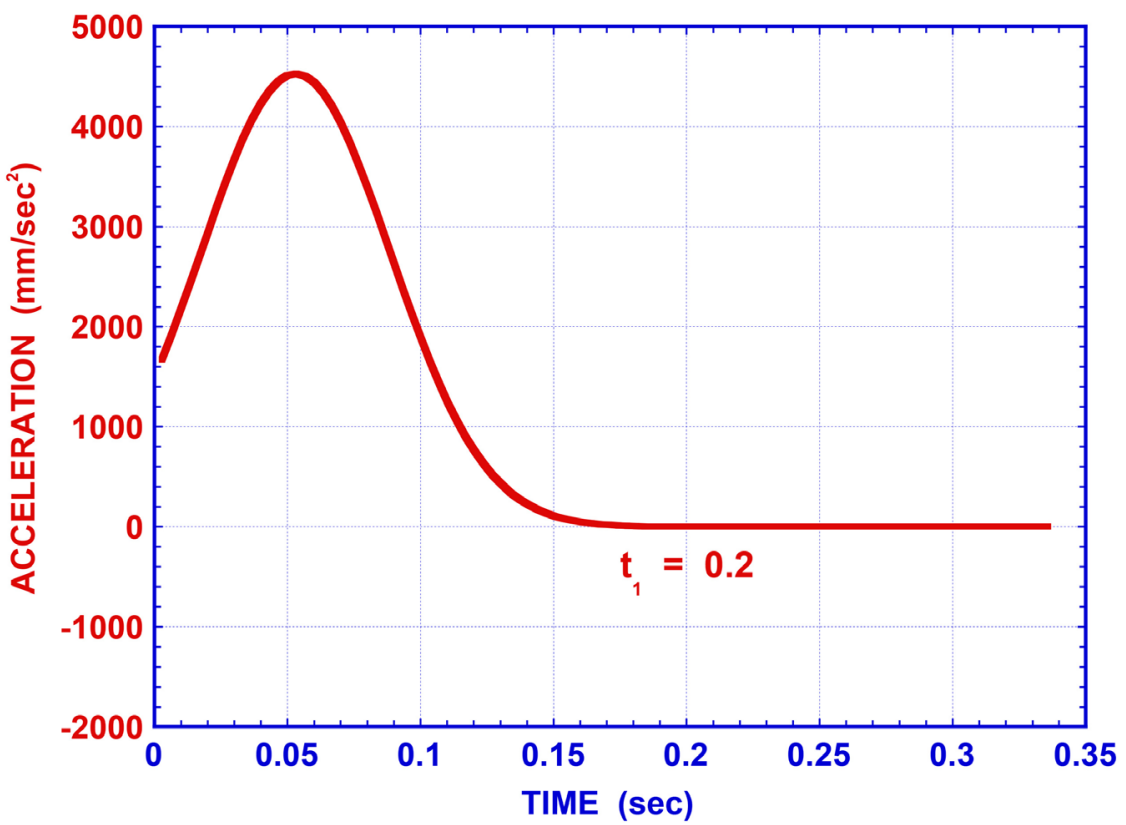

Figure 11. Dynamic experimental impulse force/acceleration [104]. 


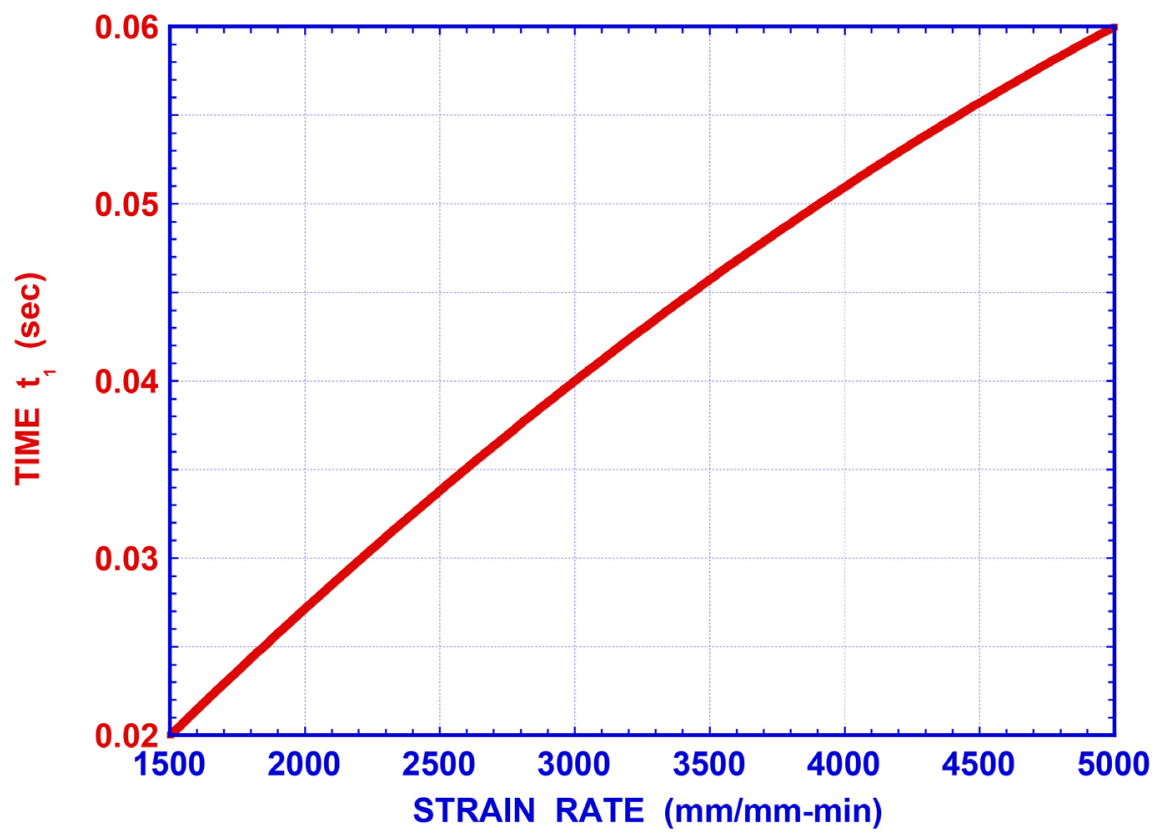

Figure 12. Time to reach steady-state strain rates $\partial \epsilon_{11}(t) / \partial t$ based on acceleration $\partial^{2} \epsilon_{11}(t) / \partial t^{2}$ measurements [104].

cannot be duplicated physically for $0 \leq t \leq \infty$ as evidenced by the typical experimental results shown in Figure 10. On the other hand, if the cross head accelerations are used then reasonable times $t_{1}$ to reach steady-state strain rates can be calculated, as seen in Figure 12. Consequently, the latter should be the protocol of choice for the determination of $t_{1}$.

Even if these two mathematical models were physically reproducible, the PRs so determined would only be applicable to the particular loading conditions under which they were experimentally determined. Consequently, the inherently specialized Class II and VI behaviors cannot be generalized and/or extended to any other loading processes since they produce process specific PRs as evidenced by Figure 1. For that matter, nor can any of the viscoelastic PR Classes I through VI be generalized as they are process specific and not universal material descriptors such as relaxation moduli and creep compliances or relaxation and creep functions.

The pervasive advantages and disadvantages of viscoelastic PRs vis-à-vis the EVCP and their lack of universality are summarized in Table 4.

\section{Conclusions}

For linear elastic and viscoelastic media:

1) Elastic PRs are of fundamental importance as linear material descriptors because they are constants for each material without regard as to what experimental protocol is used to determine their value. However, such is not the case of their linear viscoelastic counterparts as they are time, stress and stress history (path, process) dependent. Hence they have distinct values and functionality for 
each and every process. Consequently, experimentally obtained viscoelastic PRs do not match more complicated service loading conditions.

2) Elastic and viscoelastic PRs are inherently nonlinear, cf. (11) and (12). Their linear use is based on the explicit approximation of their linearity as governed by the ratio $\sigma_{11}^{E} / E^{E} \ll$.

3) All viscoelastic PRs are time dependent. Additionally, by definition PRs of all Classes, except VII, are time, stress and stress history (path, process) dependent and, therefore, non-unique, i.e. dependent on loading history. All constitutive relations involving PRs written in the time space are very computationally intensive compared to expressions involving only moduli or compliances.

4) The principal difference between PRs and moduli/compliances is that the former in all Classes except VII are an algebraic ratio of mutually perpendicular tension and/or compression strains, whereas the latter are ratios of stresses to their responding strains. This renders the PRs nonlinear functions of the two strains.

5) Unlike elastic PRs and whether in a homogeneous or non-homogeneous medium, all of the six Classes of viscoelastic Poisson's ratios are represented by functions of time, stresses and stress histories. Due to their process dependency they are non-unique material descriptors and as such unsuitable for general material characterizations. Therefore, characterizations in terms of relaxation moduli and/or creep compliances are the characterization vehicles of choice as they represent universal functions with parameters specific to each material and while

Table 4. Summary of viscoelastic PRs and moduli advantages and disadvantages.

\begin{tabular}{|c|c|c|}
\hline Class & ADVANTAGES & DISADVANTAGES \\
\hline I & none & does not lead to EVCP \\
\hline \multirow[t]{2}{*}{ II } & EVCP for & $\mathrm{PR}$ is restricted to process with \\
\hline & only $\epsilon_{11}(x)$ & a single time independent strain \\
\hline \multirow[t]{3}{*}{ III } & leads to EVCP & equivalent $\mathrm{PR}$ in time space \\
\hline & for some cases & must be properly defined \\
\hline & & as $P R$ is path dependent \\
\hline IV & none & nonlinear-no EVCP \\
\hline $\mathrm{V}$ & none & no EVCP \\
\hline \multirow[t]{2}{*}{ VI } & material dependent & no EVCP \\
\hline & but only for $\sigma_{11}(x)$ & \\
\hline \multirow[t]{2}{*}{ VII } & stress/strain independent & Eq. (32) makes use of \\
\hline & & all PRs very CPU intensive \\
\hline $\mathrm{E}, \mathrm{G}$, & stress independent unique & none \\
\hline \multirow[t]{2}{*}{$\mathrm{K}, \mathrm{C}$} & material property functions, & \\
\hline & isothermal EVCP & \\
\hline
\end{tabular}

Note: All PRs are time dependent. Additionally, by definition PRs of all Classes, except VII, are time, stress and stress history (path, process) dependent and, therefore, non-unique. All constitutive relations involving PRs written in the time space are very computationally intensive compared to expressions involving only moduli or compliances. 
time dependent they are process independent.

6) The indisputable proof of the explicit time and path dependence of viscoelastic PRs can be found in experimental results, such as those of Figure 1, where PRs due to different 1-D "constant" loading conditions are displayed. The various $\mathrm{PR}$ functions obtained from creep, relaxation and prescribed strain rate experiments bear no relations to each other and are specific and distinct expressions for each loading event.

7) Instantaneous non-zero loads, although mathematically rigorously definable by Dirac delta and/or Heaviside step functions, are physically unattainable. Consequently, the mathematically acceptable Class II and VI PR models are physically nonexistent and experimentally non-producible since they are solely based on one time independent stress or strain tensor.

8) Except in wave propagation problems, elastic loading histories are generally unimportant, since these are conservative materials without dissipation and memory. Viscoelastic materials, on the other hand, continuously dissipate energy and loading histories are of prime significance to their responses.

9) When the proper loading cycles that are physically necessary to achieve constant values of one or more state variables are included in the analyses, substantially different viscoelastic responses are achieved when compared to instantaneous loading cycles.

10) Analyses establish that Class III PRs are capable to produce EVCP protocols, on condition that physically acceptable double convolution integral constitutive relations are established (32b). However, these analyses fail to provide directions for establishing the unique, proper and necessary viscoelastic Class III PR functions of time, stress and stress history. Consequently, what process is to define the PRs "constant" strain, stress, their time derivatives, etc.? Hence, this inherent ambiguity renders viscoelastic path dependent non-unique PRs unsuited for general viscoelastic material characterization.

11) If viscoelastic PRs are to be used, then they must be obtained experimentally by loading and time processes that are exact detailed duplicates of the desired field conditions.

12) The Class VII is an artificially defined isothermal viscoelastic PR devoid of strain or stress associations and hence path independent while still remaining a time dependent universal material property descriptor. It obeys the construction mandated by Eqs. (32) necessary for the existence of EVCPs and does not suffer from the loading cycle problems associated with the other Classes. (See the next Item below.) However, its use in the time space is subject to intensive CPU use involving triple function products and double convolution integrals.

13) Since the viscoelastic PRs are time, stress and stress history dependent, unless the experimentally determined history exactly matches the application loading path no correspondence in properties can be established.

14) Some of the triple Fourier transform products, Eq. (32) necessary for establishing any PR based elastic viscoelastic correspondence principle have no physical real time counterpart constitutive relations, thus proving their nonexi- 
stence of the EVCP based on these PRs.

15) The evaluation of all the PR double convolution time integral constitutive relations stemming from the triple FT products of (32) are highly CPU intensive, thus impractical for use in analytical and/or finite element analyses. By contrast, constitutive relations without PRs but in terms of moduli or compliances lead to more efficient single convolution double product time integrals.

16) The validity of EVCPs without PRs based solely on moduli/compliances or on relaxation/creep functions remains unaffected and should be considered the universal unambiguous material characterization of choice.

17) If viscoelastic PRs are to be used in routine stress/strain analyses then their experimental determinations must exactly duplicate each and every actual service time and loading history.

18) In any viscoelastic formulation there is no need for the inclusion of PRs as all constitutive relation material properties (isotropic and anisotropic, homogeneous and nonhomogeneous, linear and nonlinear) can be unambiguously characterized by their moduli and/or compliances thus removing any controversial descriptions due to the viscoelastic $P R$ 's time and loading history dependence.

19) Finally, elastic solutions that are heavily dependent on PRs, such as thin plates, shells [129], Timoshenko beams [130], are best recast in their viscoelastic reincarnations solely in terms of relaxation moduli or creep compliances.

\section{References}

[1] Greaves, G.N., Greer, A.J., Roderic, S.L. and Rouxel, T. (2011) Poisson's Ratio and Modern Materials. Nature Materials, 10, 823-837. https://doi.org/10.1038/nmat3134

[2] Poisson, S.D. (1811) Traité de méchanique. Courcier, Paris.

[3] Poisson, S.D. (1829) Mémoire sur l'équilibre et le Mouvement des Corps Élastiques. Mémoires de P Académie Royal des Sciences de P Institut de France, 8, 357-570.

[4] Baron, K. and Thomson, W. (1865) On the Elasticity and Viscosity of Metals. Proceedings of the Royal Society, 14, 289-297. https://doi.org/10.1098/rspl.1865.0052

[5] Maxwell, J.C. (1867) On the Dynamical Theory of Gases. Philosophical Transactions of the Royal Society of London, A157, 49-88.

https://doi.org/10.1098/rstl.1867.0004

[6] Boltzmann, L.E. (1874) Zur Theorie der Elastischen Nachwirkung. Sitzungsberichte Kaiserliche Akademie Wissenhaft Wien Mathematische-Naturwissenhaft, 70, 275 306.

[7] Voigt, W. (1887) Theoretischen Studien über das Elasticitatsverhaltnisse der Kristalle. Abhandlung Geselschaft der Wissenschaft zu Göttingen, 34, 52-79.

[8] Voigt, W. (1889) Über Innere Reibung der Festen Körper, Insbesondere der Kristalle. Abhandlung der Königlichen Geselschaft der Wissenschaft zu Göttingen, 36, 1.

[9] Voigt, W. (1892) Über Innere Reibung der Festen Körper, Insbesondere der Metalle. Annallen der Physik, 47, 671-639. https://doi.org/10.1002/andp.18922831210

[10] Volterra, V. (1909) Sulle Equazioni Integrodifferenzialli Dela Teoria Dell Elastic. Atti Reale Accademia Nazionale dei Lincei, 18, 295-301. 
[11] Volterra, V. Tomassetti, M. and. Zarlatti, F.S (1913) Leçons sur les Équations Integral et les Équations Integro-Differentielles. Guthier-Villars, Paris.

[12] Turner, A.J. (1944) Non-Homogeneous Stress in Viscoelastic Media. Quarterly of Applied Mathematics, 2, 113-119. https://doi.org/10.1090/qam/10499

[13] Turner, A.J. and Doty, P. (1945) The Methods of Specifying Properties of Viscoelastic Materials. Journal of Applied Physics, 16, 700-713. https://doi.org/10.1063/1.1707524

[14] Turner, A.J. (1948) Mechanical Behavior of High Polymers. Interscience Publishers Inc., New York.

[15] Sneddon, I.N. (1951) Fourier Transforms. McGraw-Hill, New York.

[16] Read, W.T. (1950) Stress Analysis for Compressible Viscoelastic Materials. Journal of Applied Physics, 21, 671-674. https://doi.org/10.1063/1.1699729

[17] Freudenthal, A.M. (1950) The Inelastic Behavior of Engineering Materials and Structures. John Wiley \& Sons, New York.

[18] Aklonis, J.J., MacKnight, W.J. and Shen, M. (1972) Introduction to Polymer Viscoelasticity. John Wiley \& Sons, New York.

[19] Aklonis, J.J. and MacKnight, W.J. (1983) Introduction to Polymer Viscoelasticity. John Wiley \& Sons, New York.

[20] Barnes, H.A., Hutton, J.F. and Walters, K. (1989) An Introduction to Rheology. Elsevier, Amsterdam.

[21] Bartenev, G.M. and Yurri, S.Z. (1968) Strength and Failure of Viscoelastic Materials. Pergamon Press, Oxford.

[22] Bažant, Z.P., Ed. (1988) Mathematical Modeling of Creep and Shrinkage of Concrete. John Wiley and Sons, New York.

[23] Bažant, Z.P. and Luigi, C. (1991) Stability of Structures-Elastic, Inelastic, Fracture and Damage Theories. Oxford University Press, London.

[24] Bergen, J.T. (1960) Viscoelasticity: Phenomenological Aspects. Academic Press, New York.

[25] Betten, J. (2005) Creep Mechanics. 2nd Edition, Springer, New York.

[26] Biot, M.A. (1965) Mechanics of Incremental Deformations. John Wiley \& Sons, New York.

[27] Bland, D.R. (1960) The Theory of Linear Viscoelasticity. Pergamon Press, New York.

[28] Brinson, H.F. and Brinson, L.C. (2008) Polymer Engineering Science and Viscoelasticity: An Introduction. Springer, New York. https://doi.org/10.1007/978-0-387-73861-1

[29] Christensen, R.M. (1982) Theory of Viscoelasticity-An Introduction. 2nd Edition, Academic Press, New York.

[30] Drozdov, A.D. and Kolmanovski, V.B. (1994) Stability in Viscoelasticity. NorthHolland, Amsterdam.

[31] Drozdov, A.D. (1996) Finite Elasticity and Viscoelasticity-A Course in Nonlinear Mechanics of Solids. World Scientific, Singapore. https://doi.org/10.1142/2905

[32] Drozdov, A.D. (1998) Mechanics of Viscoelastic Solids. John Wiley \& Sons, New York.

[33] Drozdov, A.D. (1998) Viscoelastic Structures Mechanics of Growth and Aging. Academic Press, San Diego.

[34] Eringen, A.C. (1962) Nonlinear Theory of Continuous Media. McGraw-Hill, New 
York.

[35] Fabrizio, M. and Angelo, M. (1992) Mathematical Problems in Linear Viscoelasticity. SIAM, Philadelphia. https://doi.org/10.1137/1.9781611970807

[36] Ferry, J.D. (1980) Viscoelastic Properties of Polymers. John Wiley \& Sons, New York.

[37] Findley, W.N., Lai, J.S. and Kasif, O. (1976) Creep and Relaxation of Nonlinear Materials. North-Holland Publication Co., Amsterdam.

[38] Flügge, W. (1967) Viscoelasticity. Waltham Mass Blaisdell Publication Co., Waltham.

[39] Fung, Y.C. (1965) Foundations of Solid Mechanics. Prentice-Hall, Englewood.

[40] Luigi, G. and Onah, H.N. (1996) Viscoelastic Material Damping Technology. Becchis Osiride, Turin.

[41] Golden, J.M. and Graham, C.A.C. (1988) Boundary Value Problems in Linear Viscoelasticity. Springer Verlag, Berlin. https://doi.org/10.1007/978-3-662-06156-5

[42] Bernhard, G. (1953) Mathematical Structure of the Theories of Viscoelasticity. Hermann \& Cie, Paris.

[43] Gunasekaran, S. and Mehmet, M.A.K. (2003) Cheese Rheology and Texture. CRC Press, Boca Raton.

[44] Gurtin, M.E. and Sternberg, E. (1962) On the Linear Theory of Viscoelasticit. Archive for Rational Mechanics and Analysis, 11, 291-356. https://doi.org/10.1007/BF00253942

[45] Haddad, Y.M. (1995) Viscoelasticity of Engineering Materials. Chapman \& Hall, London. https://doi.org/10.1007/978-94-011-1272-7

[46] Hilton, H.H. (1964) An Introduction to Viscoelastic Analysis and Engineering Design for Plastics Reinhold Publishing Corp., New York, 199-276.

[47] Hilton, H.H. and Stanley, B.D. (1964) An Analogy for Anisotropic, Nonhomogeneous, Linear Viscoelasticity Including Thermal Stresses. Development in Mechanics. Pergamon Press, New York, 58-73.

[48] Huilgol, R.R. and Phan-Thien, N. (1997) Fluid Mechanics of Viscoelasticity: General Principles, Constitutive Modelling, Analytical and Numerical Techniques. Elsevier, Amsterdam.

[49] Huilgol, R.R. (1975) Continuum Mechanics of Viscoelastic Liquids. John Wiley \& Sons, New York.

[50] Hunter, S.C. (1960) Viscoelastic Waves. Progress in Solid Mechanics, 1, 3-57.

[51] Joseph, D.D. (1990) Fluid Dynamics of Viscoelastic Liquids. Springer-Verlag, New York. https://doi.org/10.1007/978-1-4612-4462-2

[52] Lakes, R.S. (1998) Viscoelastic Solids. CRC Press, Boca Rotan.

[53] Lakes, R.S. (2009) Viscoelastic Materials. Cambridge University Press, New York. https://doi.org/10.1017/CBO9780511626722

[54] Lazan, B.J. (1968) Damping of Materials and Members in Structural Mechanics. Pergamon Press, Oxford.

[55] Le Tallec, P. (1990) Numerical Analysis of Viscoelastic Problems. Springer-Verlag, Berlin.

[56] Franco, L. and Pizzetti, G. (1951) Fluage, Plasticité, Précontrainte. Dunod, Paris.

[57] Lockett, F.J. (1972) Nonlinear Viscoelastic Solids. Academic Press, London.

[58] Lodge, A.S. (1974) Body Tensor Fields in Continuum Mechanics with Applications 
to Polymer Rheology. Academic Press, New York.

[59] Lodge, A.S., Renardy, M. and Nohel, J.A. (1985) Viscoelasticity and Rheology. Academic Press, New York.

[60] Francesco, M. (2010) Fractional Calculus and Waves in Linear Viscoelasticity: An Introduction to Mathematical Models. Imperial College Press, London.

[61] Marques, S.P.C. and Guillermo, J.C. (2012) Computational Viscoelasticity. Springer, Berlin. https://doi.org/10.1007/978-3-642-25311-9

[62] McCrum, N.G., Read, B.E. and Williams, G. (1967) Anelastic and Dielectric Effects in Polymeric Solids. John Wiley \& Sons, New York.

[63] Nashif, A.D., Jones, I.G. and Henderson, J.P. (1985) Vibration Damping. John Wiley \& Sons, New York.

[64] Pipkin, A.C. (1972) Lectures on Viscoelasticity Theory. Springer-Verlag, Berlin. https://doi.org/10.1007/978-1-4615-9970-8

[65] Marcus, R. (1969) Deformation, Strain and Flow: An Elementary Introduction to Rheology. H. K. Lewis, London.

[66] Marcus, R. (1971) Advanced Rheology. H. K. Lewis, London.

[67] Renardy, M., Hrusa, W.J. and John, A.N. (1987) Mathematical Problems in Viscoelasticity. Longmans Scientific and Technical Press, Burnt Mill.

[68] Roland, C.M. (2011) Viscoelastic Behavior of Rubbery Materials. Oxford University Press, Oxford. https://doi.org/10.1093/acprof:oso/9780199571574.001.0001

[69] Salençon, J. (1983) Viscoélasticité. ENPC Presses, Paris.

[70] Schowalter, W.R. (1978) Mechanics of Non-Newtonian Fluids. Pergamon, Oxford.

[71] Scott-Blair, G.W. (1949) Survey of General and Applied Rheology. Pitman and Son, London.

[72] Shaw, M.T. and MacKnight, W.J. (2005) Introduction to Polymer Viscoelasticity, 3rd Edition, Wiley-Interscience, Hoboken. https://doi.org/10.1002/0471741833

[73] Spencer, A.J.M. (1980) Continuum Mechanics. Longman, London.

[74] Tschoegl, N.W. (1989) The Phenomenological Theory of Linear Viscoelastic Behavior: An Introduction. Springer, New York. https://doi.org/10.1007/978-3-642-73602-5.

[75] Vinogradov, G.V. and Malkin, A.Y. (1980) Rheology of Polymers: Viscoelasticity and Flow of Polymers. Mir Publishers, Moscow. https://doi.org/10.1007/978-3-642-52204-8

[76] Bertilsson, H., Delin, M., Kubat, J., Rychwalski, W.R. and Kubat, M.J. (1993) Strain Rates and Volume Changes during Short Term Creep Time of PC and PMMA. Rheologica Acta, 32, 361-369.

[77] Wineman, A.S. and Rajakopal, K.R. (2000) Mechanical Response of Polymers-An Introduction. Cambridge, New York.

[78] Clarence, Z. (1948) Elasticity and Anelasticity of Metals. University of Chicago Press, Chicago.

[79] Lakes, R.S. (1992) The Time-Dependent Poisson's Ratio of Viscoelastic Materials Can Increase or Decrease. Cellular Composites, 11, 466-469.

[80] Tschoegl, N.W., Knauss, W.G. and Emri, I. (2002) Poisson's Ratio in Linear Viscoelasticity-A Critical Review. Mechanics of Time-Dependent Materials, 6, 3-51. https://doi.org/10.1023/A:1014411503170

[81] Tschoegl, N.W. (1997) Time Dependence in Material Properties: An Overview. Mechanics of Time-Dependent Materials, 1, 3-31. 
[82] Lakes, R.S. and Wineman, A.S. (2006) On Poisson's Ratio in Linearly Viscoelastic Solids. Journal of Elasticity, 85, 45-63. https://doi.org/10.1007/s10659-006-9070-4

[83] Lakes, R.S. (1987) Foam Structures with a Negative Poisson's Ratio. Science, 235, 1038-1040. https://doi.org/10.1126/science.235.4792.1038

[84] Chen, C. P. and Lakes, R.S. (1993) Viscoelastic Behaviour of Composite Materials with Conventional-or Negative Poisson's Ratio Foam as One Phase. Journal of Materials Science, 28, 4288-4299. https://doi.org/10.1007/BF01154934

[85] Khan, K.A. and Hilton, H.H. (2010) On Inconstant Poisson's Ratios in Nonhomogeneous Elastic Media. Journal of Thermal Stresses, 33, 1-8.

[86] Hilton, H.H. (1996) On the Inadmissibility of Separation of Variable Solutions in Linear Anisotropic Viscoelasticity. International Journal of Mechanics of Composite Materials and Structures, 3, 97-100. https://doi.org/10.1080/10759419608945857

[87] Hilton, H.H. and Sung, Y. (1998) The Significance of Anisotropic Viscoelastic Poisson Ratio Stress and Time Dependencies. International Journal of Solids and Structures, 35, 3081-3095. https://doi.org/10.1016/S0020-7683(97)00357-0

[88] Hilton, H.H. (2001) Implications and Constraints of Time Independent Poisson Ratios in Linear Isotropic and Anisotropic Viscoelasticity. Journal of Elasticity, 63, 221-251.

[89] Hilton, H.H. (2009) The Elusive and Fickle Viscoelastic Poisson's Ratio and Its Relation to the Elastic-Viscoelastic Correspondence Principle. Journal of Mechanics of Materials and Structures, 4, 1341-1364.

[90] Hilton, H.H. (2011) Clarifications of Certain Ambiguities and Failings of Poisson's Ratios in Linear Viscoelasticity. Journal of Elasticity, 104, 303-318. https://doi.org/10.1007/s10659-010-9296-Z

[91] Hilton, H.H. (2011) Equivalences and Contrasts between Thermo-Elasticity and Thermo-Viscoelasticity: A Comprehensive Critique. Journal of Thermal Stresses, 34, 488-535. https://doi.org/10.1080/01495739.2011.564010

[92] Hilton, H.H. and Sung, Y. (1992) Analytical Formulation of Optimum Material Properties for Viscoelastic Damping. Journal of Smart Materials and Structures, 1,113-122.

[93] Beldica, C.E. and Hilton, H.H. (1999) Analytical Simulations of Optimum Anisotropic Linear Viscoelastic Damping Properties. Journal of Reinforced Plastics and Composites, 18, 1658-1676.

[94] Gimbel, J.A., Starver, J.J. and Soslowsky, L.J. (2004) The Effect of Overshooting the Target Strain on Estimating Viscoelastic Properties from Stress Relaxation Experiments. Journal of Biomechanical Engineering, 126, 844-848. https://doi.org/10.1115/1.1824132

[95] Beldica, C.E. and Hilton, H.H. (2012) Analytical and Computational Simulations of Experimental Determinations of Deterministic and Random Linear Viscoelastic Constitutive Relations. Proceedings of the Twelfth International Conference on Composite Materials, Paris, 5-9 July 1999, 346-371.

[96] Beldica, C.E. and Hilton, H.H. and Cyrille G. (2001) The Relation of Experimentally Generated Wave Shapes to Viscoelastic Material Characterizations-Analytical and Computational Simulations. Proceedings of the Sixteenth Annual Technical Conference of the American Society for Composites, Blacksburg, 10-12 September 2001, $1-11$.

[97] Shtark, A., Hagay G., Sameach, G. and Hilton, H.H. (2007) An Alternate Protocol for Determining Viscoelastic Material Properties Based on Tensile Tests without 
Use of Poisson Ratios. Proceedings of the 2007 International Mechanical Engineering Congress and Exposition, Seattle, 11-15 November 2007.

[98] Abudushalamu, A., Vandamme, M., Torrenti, J.M. and Benoit, M. (2015) Theoretical and Practical Differences between Creep and Relaxation Poisson's Ratios in Linear Viscoelasticity. Mechanics of Time-Dependendent Materials, 19, 537-555. https://doi.org/10.1007/s11043-015-9277-5

[99] Qazi, A., Ozer, H., Imad, L.A.Q. and Hilton, H.H. (2017) Viscoelastic and Poisson's Ratio Characterization of Asphalt Materials: Critical Review and Numerical Simulations. Materials and Structures, 50, 1-12.

[100] Abraham, S., Grosbein, H. and Hilton, H.H. (2009) Analytical Determination without Use of Poisson Ratios of Temperature Dependent Viscoelastic Material Properties Based on Uniaxial Tensile Experiments. Proceedings of the 2009 International Mechanical Engineering Congress and Exposition, Lake Buena, 13-19 November 2009.

[101] Michaeli, M., Abraham, S., Grosbein, H. and Hilton, H.H. (2010) A Computational Protocol for the Alternative Determination of Viscoelastic Material Properties without Poisson's Ratios. Proceedings Times of Polymers and Composites, 1255, 37-39.

[102] Shtark, A., Grosbein, H. and Hilton, H.H. (2012) An Alternate Protocol for Determining Viscoelastic Material Properties Based on Tensile Tests without Use of Poisson Ratios. Journal of Applied Mechanics, 8, 1361.

[103] Michaeli, M., Shtark, A., Grosbein, H. and Hilton, H.H. (2012) Characterization of Isotropic Viscoelastic Moduli and Compliances from 1-D Tension Experiments. Proceedings of the Fifty-Third AIAA/ASME/ASCE/AHS/ASC Structures, Structural Dynamics and Materials (SDM) Conference, Waikiki, 23-26 April 2012, 1547. https://doi.org/10.2514/6.2012-1547

[104] Michaeli, M., Shtark, A., Grosbein, H., Altus, E. and Hilton, H.H. (2013) A Unified Approach to Characterization of Isotropic Linear Viscoelastic Moduli and Compliances Directly from 1-D Tensile Experiments. Proceedings of the Fifty-fourth AIAA/ASME/ASCE/AHS/ASC Structures, Structural Dynamics and Materials (SDM) Conference, 1692.

[105] Turner, S.R. (1973) Creep in Glassy Polymers. In: Holliday, L., Kelly, A. and Haward, R.N., Eds. The Physics of Glassy Polymers, John Wiley \& Sons, New York, 223-278. https://doi.org/10.1007/978-94-010-2355-9_5

[106] Sneddon, I.N. (1961) Special Functions of Mathematical Physics and Chemistry. Oliver and Boyd, London.

[107] Schapery, R.A. (1969) On the Characterization of Nonlinear Viscoelastic Materials. Polymer Engineering Science, 9, 295-310. https://doi.org/10.1002/pen.760090410

[108] Knauss, W.G. and Zhao, J. (2007) Improved Relaxation Time Coverage in RampStrain Histories. Mechanics of Time-Dependent Materials, 11, 199-216.

[109] Tscharnuter, D., Jerabek, M., Zoltan, M. and Reinhold, W.L. (2011) On the Determination of the Relaxation Modulus of PP Compounds Including Ramp Strain Loading. Mechanics of Time-Dependendent Materials, 15, 1-14.

https://doi.org/10.1007/s11043-010-9119-4

[110] Tscharnuter, D., Jerabek, M. Zoltan, M. and Reinhold, W.L. (2011) Time Dependent Poisson's Ratio of Polypropylene Compounds for Various Strain Histories. Mechanics of Time-Dependent Materials, 15, 15-28.

https://doi.org/10.1007/s11043-010-9121-x

[111] Luigi, G. and D'Amore, A. (2009) The Relative Placement of Linear Viscoelastic 
Functions in Amorphous Glassy Polymers. Journal of Rheology, 53, 339-356.

[112] Luigi, G., D'Amore, A. and Simon, S.L. (2010) On the Viscoelastic Poisson's Ratio in Amorphous Polymers. Journal of Rheology, 54, 1009-1022.

[113] Emad, K., Grasley, Z.C. and Masad, E. (2013) Viscoelastic Poisson's Ratio of Asphalt Mixtures. International Journal of Geomechanics, 13, 162-169.

[114] Erin, A., Li, R.Z. and Chew, H.B. (2013) Negative Stiffness Induced by Shear along Wavy Interfaces. Journal of Mechanics and Physics of Solids.

[115] Guo, J.X., Luigi, G. and Simon, S.L. (2012) Bulk and Shear Rheology of a Symmetric Three-Arm Star Polystyrene. Journal of Polymer Science Part B: Polymer Physics, 50, 1233-1244. https://doi.org/10.1002/polb.23113

[116] Churchill, R.V. (1944) Modern Operational Mathematics in Engineering. McGrawHill Book Co., New York.

[117] Sokolnikoff, I.S. (1956) Mathematical Theory of Elasticity. McGraw-Hill, New York.

[118] Chou, P.C. and Pagano, N.J. (1992) Elasticity-Tensor Dyadic, and Engineering Approaches. Dover, New York.

[119] Timoshenko, S.P. (1934) Theory of Elasticity. McGraw-Hill, New York.

[120] Love, A.E.H. (1944) A Treatise on the Mathematical Theory of Elasticity. Dover Publications, New York.

[121] Mardsden, J.E. and Thomas, R.H. (1983) Mathematical Foundation of Elasticity. Dover, New York.

[122] Prony, C., Gaspard, F.M. and De Baron, R. (1795) Essai Experimental et Analytique. Journal de l'École Polytechnique de Paris, 1, 24-76.

[123] Poularikas, A.D. (2011) Transforms and Applications Handbook, 3rd Edition, CRC Press, Boca Raton.

[124] Hilton, H.H. and Russell, H.G. (1961) An Extension of Alfrey's Analogy to Thermal Stress Problems in Temperature Dependent Linear Viscoelastic Media. Journal of Physics and Solids, 9, 152-164. https://doi.org/10.1016/0022-5096(61)90014-X

[125] Hilton, H.H. and Clements, J.R. (1964) Formulation and Evaluation of Approximate Analogies for Temperature Dependent Linear Viscoelastic Media. Thermal Loading and Creep, 6, 17-24.

[126] Dirac, P.A.M. (1958) Quantum Mechanics. 4th Edition, Oxford University Press, London.

[127] Hilton, H.H. (2016) Nonlinear Elastic and Viscoelastic 1-D Wave Propagation Modeling and Analysis. International Journal of Mathematics in Engineering, Science and Aerospace, 8, 301-332.

[128] Raymond, B.L., Ashley, H. and. Halfman, R.L. (1955) Aeroelasticity. Dover Publications, New York.

[129] Timoshenko, S.P. (1940) Theory of Plates and Shells. McGraw-Hill, New York.

[130] Hilton, H.H. (2009) Viscoelastic Timoshenko Beam Theory. Mechanics of TimeDependent Materials, 13, 1-10. https://doi.org/10.1007/s11043-008-9075-4 
Submit or recommend next manuscript to SCIRP and we will provide best service for you:

Accepting pre-submission inquiries through Email, Facebook, LinkedIn, Twitter, etc. A wide selection of journals (inclusive of 9 subjects, more than 200 journals)

Providing 24-hour high-quality service

User-friendly online submission system

Fair and swift peer-review system

Efficient typesetting and proofreading procedure

Display of the result of downloads and visits, as well as the number of cited articles Maximum dissemination of your research work

Submit your manuscript at: http://papersubmission.scirp.org/

Or contact msa@scirp.org 